\title{
TOTALLY RAMIFIED VALUATIONS ON FINITE-DIMENSIONAL DIVISION ALGEBRAS
}

\author{
J.-P. TIGNOL ${ }^{1}$ AND A. R. WADSWORTH ${ }^{2}$
}

\begin{abstract}
Division algebras $D$ with valuation $v$ are studied, where $D$ is finite-dimensional and totally ramified over its center $F$ (i.e., the ramification index of $v$ over $\left.v\right|_{F}$ equals $[D: F]$ ). Such division algebras have appeared in some important constructions, but the structure of these algebras has not been systematically analyzed before. When $\left.v\right|_{F}$ is Henselian a full classification of the $F$-subalgebras of $D$ is given. When $F$ has a Henselian valuation $v$ with separably closed residue field and $A$ is any tame central simple $F$-algebra, an algorithm is given for computing the underlying division algebra of $A$ from a suitable subgroup of $A^{*} / F^{*}$. Some examples are constructed using this valuation theory, including the first example of finite-dimensional $F$-central division algebras $D_{1}$ and $D_{2}$ with $D_{1} \otimes_{F} D_{2}$ not a division ring, but $D_{1}$ and $D_{2}$ having no common subfield $K \supsetneqq F$.
\end{abstract}

Valuation theory, long a basic tool in commutative algebra, has been relatively neglected in the study of division algebras, until quite recently. Nontheless, valuations are naturally present in a number of division algebras that have been constructed to exhibit special properties, particularly algebras over iterated Laurent power series fields. For example, such division algebras have been key ingredients in Amitsur's noncrossed product construction [Am] and in Platonov's construction $[\mathbf{P}]$ of division algebras $D$ with $S K_{1}(D) \neq 1$. Valuations are not so prevalent on division algebras as on fields. But if a division algebra $D$ does have a valuation, this structure contains a substantial amount of information about $D$ which would scarcely be accessible otherwise.

We consider here valued division algebras $D$ for which $D$ is totally ramified and tame over its center $F$, i.e., for which the ramification index $\left|\Gamma_{D}: \Gamma_{F}\right|$ equals the dimension $[D: F]$ of $D$ over $F$ and the characteristic $\operatorname{char}(\bar{D})$ does not divide $[D: F]$. (Here $\Gamma_{D}$ is the value group of the valuation on $D$, and $\bar{D}$ is the residue division algebra. We assume throughout that $[D: F]<\infty$.) Valued division algebras of this type appear, e.g., in Amitsur's noncrossed product paper [Am, §2], in Saltman's work on indecomposable division algebras [Sa], in certain of the MalcevNeumann division algebras considered by the first author and Amitsur [ $\left.\mathbf{T} \mathbf{A}_{\mathbf{2}}, \S 4\right]$, etc. However, the intrinsic structure of totally ramified tame division algebras has apparently not been examined closely before. This may be because most past work on valued division algebras has concentrated on discrete valuations, when $\Gamma_{D} \cong \mathbf{Z}$; for such a valuation $D$ is never totally ramified over its center $F$ unless $D=F$ (cf. (3.2) below).

Received by the editors June 26, 1986.

1980 Mathematics Subject Classification (1985 Revision). Primary 16A39.

${ }^{2}$ Supported in part by F.N.R.S.

${ }^{2}$ Supported in part by the National Science Foundation. 
If $D$ is tame and totally ramified over its center $F$ with respect to a valuation $v$, we will prove that there is a natural nondegenerate symplectic pairing on the relative value group $\Gamma_{D} / \Gamma_{F}$. We will show how this pairing limits the possible decompositions of $D$ into tensor products of cyclic algebras (Proposition 3.3) and will show (Theorem 3.8) that if $v$ is Henselian on $F$, then the subgroups of $\Gamma_{D} / \Gamma_{F}$ completely classify the isomorphism classes of $F$-subalgebras of $D$. If $(F, v)$ is strictly Henselian (i.e., Henselian with $\bar{F}$ separably closed), we provide in $\S 4$ an algorithm for determining the underlying division algebra of a central simple $F$ algebra. Among other things, this allows one to calculate the underlying division algebra of a tensor product of two $F$-central division algebras (Example 4.4(i)). Every tame division algebra $D$ over such an $F$ is necessarily totally ramified, and our algorithm allows a full determination of which fields algebraic over $F$ split $D$ (Proposition 4.5).

The main results on totally ramified tame division algebras are given in $\S \S 3-4$, with preliminary machinery and terminology set up in $\S \S 1-2$. In $\S 5$ we apply the general theory to a couple of examples. Notably, we give an example of division algebras $D_{1}$ and $D_{2}$ with center $F$ such that $D_{1} \otimes_{F} D_{2}$ is not a division ring but there is no proper extension field of $F$ lying in both $D_{1}$ and $D_{2}$. This settles an old question in the theory of algebras.

1. Valued division algebras. In this section we establish basic terminology and recall some of the essential facts about valuations on division rings and fields that will be used frequently. We also prove a convenient extension lemma for a valued division algebra totally ramified over its center.

Every ring $A$ considered here is assumed to be a finite-dimensional algebra over some field. ("Field" means commutative field.) We assume always that $A$ has a 1 , and write $A^{*}$ for the group of units of $A$. The center of $A$ is denoted $Z(A)$. We write $|S|$ for the cardinality of a set $S$.

Let $D$ be a division ring. A valuation on $D$ is a function $v: D^{*} \rightarrow G$, where $G$ is a totally ordered abelian group (written additively), such that for all $a, b \in D^{*}$,

(i) $v(a b)=v(a)+v(b)$;

(ii) $v(a+b) \geq \min (v(a), v(b))$ if $b \neq-a$.

The standard reference for valuation theory on division rings is Schilling's book [S]. (Schilling does not require the ordered group $G$ to be abelian. However, since we are assuming that $D$ is a finite-dimensional algebra over a field an easy argument (cf. $\left[\mathbf{W}_{\mathbf{2}}\right]$ ) shows that $v\left(D^{*}\right)$ is abelian even if $G$ is not abelian.) Let $\Gamma_{D}:=v\left(D^{*}\right)$, the value group of $v$ on $D ; V_{D}:=\left\{a \in D^{*} \mid v(a) \geq 0\right\} \cup\{0\}$, the valuation ring of $v$; $M_{D}:=\left\{a \in D^{*} \mid v(a)>0\right\} \cup\{0\}$, the unique maximal left ideal and maximal right ideal of $V_{D} ; U_{D}:=V_{D}-M_{D}=V_{D}^{*}$, the group of $v$-units; and $\bar{D}:=V_{D} / M_{D}$, the residue division ring of $v$. When (not until §5) we consider more than one valuation on the same division ring, we will write $\Gamma_{D, v}, \ldots, V_{D, v}, \bar{D}_{v}$. For $a \in V_{D}$ we write $\bar{a}$ for the image of $a$ in $\bar{D}$.

Let $E$ be any division ring which is a subring of the valued division $\operatorname{ring}(D, v)$. The restriction $\left.v\right|_{E}$ of $v$ to $E^{*}$ is clearly a valuation of $E$. Then $\Gamma_{E}$ is a subgroup of $\Gamma_{D}$ and $\left|\Gamma_{D}: \Gamma_{E}\right|$ is the ramification index of $D$ over $E$ with respect to $v$. Also $V_{D} \cap E=V_{E}$ and $M_{D} \cap E=M_{E}$, so we can view $\bar{E}$ as a subring of $\bar{D}$. The residue degree of $D$ over $E$ is $[\bar{D}: \bar{E}]$, which is the dimension of $\bar{D}$ as a left $\bar{E}$-vector space. 
Recall (e.g from [S, pp. 21-22]) the "fundamental inequality"

$$
\left|\Gamma_{D}: \Gamma_{E}\right| \cdot[\bar{D}: \bar{E}] \leq[D: E] .
$$

$D$ is said to be "defectless" over $E$ if $[D: E]<\infty$ and equality holds in (1.1). (For example, it can be shown that if $\Gamma_{D} \cong \mathbf{Z}$, then $D$ is defectless over $Z(D)$. We omit the proof, since we will have no occasion to use this fact.) Assume $[D: E]<\infty$. We say that $D$ is totally ramified over $E$ with respect to $v$ if $\left|\Gamma_{D}: \Gamma_{E}\right|=[D: E]$. When this occurs, (1.1) shows that $\bar{D}=\bar{E}$. At the other extreme, if $[\bar{D}: \bar{E}]=[D: E]$ we say that $D$ is inertial over $E$. Finally, we say $D$ is tame over $E$ if $\operatorname{char}(\bar{E}) \nmid$ $[D: E]$. In his posthumous paper $\left[\mathbf{D}_{\mathbf{2}}\right]$ P. Draxl proved a "noncommutative Ostrowski theorem," which is a noteworthy refinement of (1.1). Draxl's theorem $\left[\mathbf{D}_{\mathbf{2}}\right.$, Theorem 2] says: If the restriction of $v$ to $Z(E)$ is Henselian, then

$$
[D: E]=[\bar{D}: \bar{E}] \cdot\left|\Gamma_{D}: \Gamma_{E}\right| \cdot p^{b},
$$

where $p=\operatorname{char}(\bar{E})(p=1$ if $\operatorname{char}(\bar{E})=0)$.

Given division rings $E \subseteq D$, suppose $v$ is a valuation on $E$. We say that $v$ extends to $D$ if there is a valuation $w$ on $D$ with $\left.w\right|_{E}=v$. When $v$ has a unique extension to $D$ (i.e., unique up to the choice of $\Gamma_{D} \supseteq \Gamma_{E}$ ) we will routinely use the same letter $v$ for the extended valuation. Let $\Delta$ be the divisible hull of the torsion-free abelian group $\Gamma_{E}$. Then $\Delta \cong \Gamma_{E} \otimes_{\mathbf{Z}} \mathbf{Q}$, and the ordering on $\Gamma_{E}$ extends uniquely to a total ordering on $\Delta$. Suppose $[D: E]<\infty$ and $v$ extends to a valuation on $D$; then as $\Gamma_{D} / \Gamma_{E}$ is torsion and $\Gamma_{D}$ is torsion-free there is a unique monomorphism $f: \Gamma_{D} \rightarrow \Delta$ which restricts to the injection $\Gamma_{E} \hookrightarrow \Delta$, and $f$ is order-preserving. In light of this, we will view $\Gamma_{D}$ as a subgroup of $\Delta$, thereby removing the ambiguity about the choice of $\Gamma_{D} \supseteq \Gamma_{E}$.

Unlike the situation with fields, it is not always true that every valuation on the division algebra $E$ can be extended to a valuation on $D \supseteq E$. This has been a serious obstacle in the development of noncommutative valuation theory, and has led to consideration of other valuation-like conditions with better extension properties (cf. [VG]). There is a general criterion given by M. Krasner and P. M. Cohn (cf. [VG, p. 31] or [CM, Theorem 2.3]) for when a valuation can be extended from $E$ to $D$. Another criterion has been given for the special case $E=Z(D)$ (cf. $\left[\mathbf{W}_{\mathbf{2}} ; \mathbf{C}\right.$, Theorem 1]):

VALUATION EXTENSION CRITERION 1.3. Let $D$ be a division ring (with $[D: Z(D)]<\infty$, as always) and let $v$ be a valuation on $Z(D)$. Then $v$ has an extension to a valuation on $D$ if and only if $v$ extends uniquely to each field $K$ with $Z(D) \subseteq K \subseteq D$.

It is immediate from this criterion that if $v$ is any valuation on $Z(D)$, then $v$ has at most one extension to $D$. Furthermore, the criterion indicates why most noncommutative valuation theory has been carried out on division algebras over Henselian fields. For, recall $[\mathbf{E},(16.4),(16.6)]$ that a valuation $v$ on a field $F$ is Henselian if and only if $v$ has a unique extension to each field $K$ algebraic over $F$. Thus, if $(F, v)$ is Henselian and a division ring $D$ is a finite-dimensional $F$-algebra, then $v$ has a unique extension to $D$. For, $v$ extends uniquely to $Z(D)$, and $(Z(D), v)$ is also Henselian, so (1.3) applies. 
In our study of totally ramified tame division algebras we will make heavy use of the basic facts about totally ramified tame field extensions. We recall those facts below, after introducing some more terminology.

For any field $F$ and any positive integer $l$ write $\mu(F)$ for the group of roots of unity of $F$ and $\mu_{l}(F)$ for the cyclic group of $l$ th roots of unity of $F$. We write $\mu_{l} \subseteq F$ in short for: $F$ contains $l$ th roots of unity. (This can only occur if $\operatorname{char}(F) \nmid l$.) The notation $\mu_{l}(F)$ will only be used when $\mu_{l} \subseteq F$.

If $G$ is a finite abelian group, let $\hat{G}:=\operatorname{Hom}(G, \mathbf{Q} / \mathbf{Z})$, the dual group of $G$. Recall that $G \cong \hat{G}$, though not canonically. For $g \in G$, we write $\operatorname{ord}(g)$ for the order of $g$ in $G$; let $\exp (G):=\operatorname{lcm}\{\operatorname{ord}(g) \mid g \in G\}$, the exponent of $G$. If $G_{1}, G_{2}, G_{3}$ are groups with identity elements $1_{G_{i}}$, then a function $f: G_{1} \times G_{2} \rightarrow G_{3}$ is said to be nondegenerate if for each $g_{i} \in G_{i}, g_{i} \neq 1_{G_{i}}, i=1,2$, there is an $h_{j} \in G_{j}, j=3-i$, with $f\left(g_{1}, h_{2}\right) \neq 1_{G_{3}}$ and $f\left(h_{1}, g_{2}\right) \neq 1_{G_{3}}$.

Now, suppose $F \subseteq K$ are fields with $[K: F]<\infty$. Let $v$ be a valuation on $K$ with respect to which $K$ is tame and totally ramified over $F$. Let $l=\exp \left(\Gamma_{K} / \Gamma_{F}\right)$. Then, recall

Proposition 1.4. (i) Suppose $K$ is Galois over $F$, with $\mathcal{G}(K / F)$ the Galois group. Then $\mu_{l} \subseteq \bar{F}$, and there is a well-defined nondegenerate bilinear pairing

$$
\gamma:\left(\Gamma_{K} / \Gamma_{F}\right) \times \mathcal{G}(K / F) \rightarrow \mu_{l}(\bar{F}) \quad \text { given by } \gamma\left(v(c)+\Gamma_{F}, \tau\right)=\overline{c / \tau(c)} \in \bar{F}
$$

for all $c \in K^{*}$ and $\tau \in \mathcal{G}(K / F)$. The pairing induces an isomorphism $\mathcal{G}(K / F) \cong$ $\widehat{\Gamma}_{K} / \Gamma_{F}$. Hence, $\mathcal{G}(K / F)$ is an abelian group with $\mathcal{G}(K / F) \cong \Gamma_{K} / \Gamma_{F}$.

(ii) $K$ is a Kummer extension of $F$ if and only if $K$ is Galois over $F$ and $\mu_{l} \subseteq F$. When this occurs, let $S=\left\{a \in K^{*} \mid a^{l} \in F^{*}\right\}$, the Kummer set of $K$ over $F$. Then the map $\bar{v}: S / F^{*} \rightarrow \Gamma_{K} / \Gamma_{F}$ induced by $v$ is an isomorphism, and the diagram

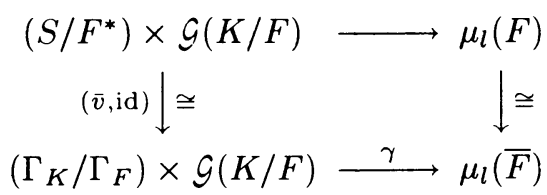

is commutative, where the upper map is the nondegenerate Kummer pairing given by $\left(a F^{*}, \tau\right) \mapsto a / \tau(a)$.

(iii) Suppose $(F, v)$ is Henselian. Then $K$ is a compositum of radical extensions of $F$ (even if $K / F$ is not Galois). Thus, $K$ is a Kummer extension if and only if $\mu_{l} \subseteq F$ if and only if $\mu_{l} \subseteq \bar{F}$.

For a proof of $(1.4)(\mathrm{i})$, see [E, (20.11), pp. 161-162]. The first sentence of (ii) is immediate from (i). The injectivity of $\bar{v}$ follows from the obvious commutativity of diagram (1.5) and the nondegeneracy of the Kummer pairing (cf. [ $\mathbf{J}_{\mathbf{1}}$, pp. 119123]). Then $\bar{v}$ is surjective since $\left|\Gamma_{K}: \Gamma_{F}\right|=[K: F]=\left|S / F^{*}\right|$. For (iii), see $[\mathbf{S}$, p. 64, Theorem 3].

Valued division algebras are often easier to work with when the valuation on the center is Henselian with sufficiently large residue field. The next lemma provides a device for reducing to that case when the division algebra is totally ramified over its center.

ExTENSION LEMMA 1.6. Let $F$ be a field with valuation $v$. Let $D_{1}$ and $D_{2}$ be division algebras over $F$ with respective valuations $w_{1}$ and $w_{2}$ extending $v$ on $F$. 
Suppose $\left[D_{1}: F\right]<\infty, D_{1}$ is totally ramified over $F$, and $\Gamma_{D_{1}} \cap \Gamma_{D_{2}}=\Gamma_{F}$. Then $D_{1} \otimes_{F} D_{2}$ is a division ring with a unique valuation $w$ extending $w_{1}$ on $D_{1} \otimes F$ and $w_{2}$ on $F \otimes D_{2}$. The value group of $w$ is $\Gamma_{D_{1}}+\Gamma_{D_{2}}$ and $D_{1} \otimes D_{2}$ is totally ramified over $D_{2}$.

Proof. Let $\Delta$ be the divisible hull of $\Gamma_{D_{2}}$. Since $\Gamma_{D_{1}} / \Gamma_{F}$ is torsion, the injection $\Gamma_{F} \hookrightarrow \Gamma_{D_{2}} \hookrightarrow \Delta$ extends uniquely to an injection $\Gamma_{D_{1}} \hookrightarrow \Delta$, which is order-preserving. In computing $\Gamma_{D_{1}} \cap \Gamma_{D_{2}}$ and $\Gamma_{D_{1}}+\Gamma_{D_{2}}$ we view $\Gamma_{D_{1}}$ as a subgroup of $\Delta$.

Choose and fix inverse images $b_{1}, \ldots, b_{k}$ in $D_{1}^{*}$ of all the distinct elements of $\Gamma_{D_{1}} / \Gamma_{F}$. Then in any linear combination $\sum_{i=1}^{k} c_{i} b_{i}$ with $c_{i} \in F$, the nonzero summands $c_{i} b_{i}$ all have different values under $w_{1}$. Thus, the $b_{i}$ are $F$-linearly independent, so (by dimension count) they form an $F$-basis of $D_{1}$. Also in any nonzero $F$-linear combination $\sum c_{i} b_{i}$, since the minimum value among the nonzero summands occurs just once, $w_{1}\left(\sum c_{i} b_{i}\right)=\inf \left\{w_{1}\left(c_{i} b_{i}\right) \mid c_{i} \neq 0\right\}$.

Every element $d \in D_{1} \otimes_{F} D_{2}$ has a unique representation $d=\sum_{i=1}^{k} b_{i} \otimes r_{i}$, with each $r_{i} \in D_{2}$. If $D_{1} \otimes D_{2}$ has a valuation $w$ extending $w_{1}$ and $w_{2}$, then for $r_{i} \neq 0$, $w\left(b_{i} \otimes r_{i}\right)=w\left(\left(b_{i} \otimes 1\right) \cdot\left(1 \otimes r_{i}\right)\right)=w_{1}\left(b_{i}\right)+w_{2}\left(r_{i}\right) \in \Delta$. Since $\Gamma_{D_{1}} / \Gamma_{F}$ injects into $\Delta / \Gamma_{D_{2}}, w\left(b_{i} \otimes r_{i}\right) \neq w\left(b_{j} \otimes r_{j}\right)$ for $i \neq j$. Thus, we must have

$$
w\left(\sum_{i=1}^{k} b_{i} \otimes r_{i}\right)=\inf _{1 \leq i \leq k}\left\{w_{1}\left(b_{i}\right)+w_{2}\left(r_{i}\right) \mid r_{i} \neq 0\right\} .
$$

We call the unique summand $b_{j} \otimes r_{j}$ with minimum value the leading term of $\sum b_{i} \otimes r_{i}$. It remains to check that formula (1.7) actually defines a valuation on $D_{1} \otimes_{F} D_{2}$. For $d=\sum_{i} b_{i} \otimes r_{i}$ and $e=\sum_{i} b_{i} \otimes s_{i} \in D_{1} \otimes D_{2}-\{0\}$ with $e \neq-d$ it is easy to see that $w(d+e) \geq \inf \{w(d), w(e)\}$; hence $w(d+e)=\inf \{w(d), w(e)\}$ if $w(d) \neq w(e)$. For the behavior of $w$ under products, consider first $\left(b_{j} \otimes r_{j}\right) \cdot\left(b_{k} \otimes s_{k}\right)$ with $r_{j}, s_{k} \neq 0$. Write $b_{j} b_{k}=\sum_{i} c_{i} b_{i}$ with the $c_{i} \in F$. Then,

$$
\begin{aligned}
w\left(\left(b_{j} \otimes r_{j}\right)\left(b_{k} \otimes s_{k}\right)\right) & =w\left(\sum_{i} b_{i} \otimes\left(c_{i} r_{j} s_{k}\right)\right) \\
& =\inf \left\{w_{1}\left(b_{i}\right)+v\left(c_{i}\right)+w_{2}\left(r_{j}\right)+w_{2}\left(s_{k}\right) \mid c_{i} \neq 0\right\} \\
& =w_{1}\left(b_{j} b_{k}\right)+w_{2}\left(r_{j}\right)+w_{2}\left(s_{k}\right) \\
& =w\left(b_{j} \otimes r_{j}\right)+w\left(b_{k} \otimes s_{k}\right) .
\end{aligned}
$$

Hence, if $d e \neq 0$,

$$
\begin{aligned}
w(d e) & =w\left(\sum\left(b_{i} \otimes r_{i}\right) \cdot \sum\left(b_{j} \otimes s_{j}\right)\right) \\
& \geq \inf \left\{w\left(\left(b_{i} \otimes r_{i}\right)\left(b_{j} \otimes s_{j}\right)\right) \mid r_{i} \neq 0, s_{j} \neq 0\right\} \\
& =\inf \left\{w\left(b_{i} \otimes r_{i}\right)+w\left(b_{j} \otimes s_{j}\right)\right\}=w(d)+w(e),
\end{aligned}
$$

i.e., $w(d e) \geq w(d)+w(e)$. Now, let $b_{j} \otimes r_{j}$ be the leading term of $d$. Then $d=\left(b_{j} \otimes r_{j}\right)+d^{\prime}$ where $d^{\prime}=0$ or $w\left(d^{\prime}\right)>w\left(b_{j} \otimes r_{j}\right)$. Likewise write $e=\left(b_{k} \otimes s_{k}\right)+e^{\prime}$ where $b_{k} \otimes s_{k}$ is the leading term of $e$. Then $d e=\left(b_{j} \otimes r_{j}\right)\left(b_{k} \otimes s_{k}\right)+\rho$, where $\rho=d^{\prime}\left(b_{k} \otimes s_{k}\right)+\left(b_{j} \otimes r_{j}\right) e^{\prime}+d^{\prime} e^{\prime}$. Now, if $\rho \neq 0, w(\rho)>w\left(\left(b_{j} \otimes r_{j}\right)\left(b_{k} \otimes s_{k}\right)\right)$ by what has already been proved. Hence, $\rho \neq-\left(b_{j} \otimes r_{j}\right)\left(b_{k} \otimes s_{k}\right)$. Therefore, $d e \neq 0$ and $w(d e)=w\left(\left(b_{j} \otimes r_{j}\right)\left(b_{k} \otimes s_{k}\right)\right)=w(d)+w(e)$. This shows that $D_{1} \otimes D_{2}$ has 
no zero divisors, and that $w$ is a valuation. Since $D_{1} \otimes D_{2}$ is finite-dimensional over $D_{2}$, hence Artinian, it must be a division ring. Clearly $\Gamma_{D_{1} \otimes D_{2}}=\Gamma_{D_{1}}+\Gamma_{D_{2}}$. Hence,

$$
\left|\Gamma_{D_{1} \otimes D_{2}}: \Gamma_{D_{2}}\right|=\left|\Gamma_{D_{1}}: \Gamma_{D_{1}} \cap \Gamma_{D_{2}}\right|=\left|\Gamma_{D_{1}}: \Gamma_{F}\right|=\left[D_{1}: F\right]=\left[D_{1} \otimes D_{2}: D_{2}\right],
$$

which shows that $D_{1} \otimes D_{2}$ is totally ramified over $D_{2}$.

REMARK. B. Fein has informed us that the special case of the extension lemma with $D_{1}$ and $D_{2}$ both finite-dimensional and totally ramified over $F$ appeared in the unpublished papers of the late P. Draxl.

2. Symplectic pairings and armatures of algebras. In considering tensor products of symbol algebras or totally ramified division algebras, certain symplectic pairings on finite abelian groups arise naturally. We now summarize the basic facts about such pairings, and then describe the pairing associated with a tensor product of symbol algebras.

Let $G$ be a finite abelian group. We use multiplicative notation for $G$ and write $1_{G}$ (or just 1) for its identity element. For $g \in G$, we write $\langle g\rangle$ for the cyclic subgroup generated by $g$. We say that $\left\{g_{1}, \ldots, g_{k}\right\}$ is a base of $G$ if $G$ is the internal direct product $G=\left\langle g_{1}\right\rangle \times \cdots \times\left\langle g_{k}\right\rangle$. Let $n_{i}=\operatorname{ord}\left(g_{i}\right), i=1,2, \ldots, k$. If $n_{2}\left|n_{1}, n_{3}\right| n_{2}, \ldots, n_{k} \mid n_{k-1}$ and $n_{k}>1$, then $n_{1}, \ldots, n_{k}$ are the invariant factors of $G$, which determine $G$ up to isomorphism. The rank of $G$ is the minimum size of a generating set of $G$, i.e., the number of invariant factors of $G$.

Let $B$ be a (bimultiplicative) symplectic pairing on $G$, that is, a function

$$
B: G \times G \rightarrow C
$$

(where $C$ is a group (written multiplicatively) isomorphic to a subgroup of $\mathbf{Q} / \mathbf{Z}$ ) such that, for all $g_{1}, g_{2}, g, h \in G$,

(i) $B\left(g_{1} g_{2}, h\right)=B\left(g_{1}, h\right) B\left(g_{2}, h\right)$;

(ii) $B\left(h, g_{1} g_{2}\right)=B\left(h, g_{1}\right) B\left(h, g_{2}\right)$;

(iii) $B(g, g)=1_{C}$.

So, $B(g, h)=B(h, g)^{-1}$ for all $g, h \in G$. Note that the image of $B$ is a finite (hence cyclic) subgroup of $C$. For any subgroup $H$ of $G$ let $H^{\perp}:=\{g \in G \mid B(g, h)=1$ for all $h \in H\}$, a subgroup of $G$. We call $H^{\perp}$ the orthogonal dual of $H$ with respect to $B$. The radical of $G, \operatorname{rad}(G)$, is defined to be $G^{\perp}$.

Assume $B$ is nondegenerate in the terminology of $\S 1$, i.e., $\operatorname{rad}(G)=\left\{1_{G}\right\}$. Then $|\operatorname{im}(B)|=\exp (G)$ and $B$ induces an isomorphism $G \cong \operatorname{Hom}(G, C)$ via $g \mapsto B(g,-)$; also, $\operatorname{Hom}(G, C) \cong \hat{G}$ as $C$ embeds in $\mathbf{Q} / \mathbf{Z}$ and $\exp (G)|| C \mid$. From the duality for finite abelian groups we have, for any subgroup $H$ of $G$,

$$
G / H^{\perp} \cong \operatorname{Hom}(H, C) \cong \hat{H} \cong H,
$$

$$
\left|H^{\perp}\right| \cdot|H|=|G| \text { and } \quad H^{\perp \perp}=H .
$$

Note that the restriction of $B$ to $H \times H$ is a symplectic pairing on $H$ whose radical is $H \cap H^{\perp}$. Thus, $B$ induces a nondegenerate symplectic pairing on $H /\left(H \cap H^{\perp}\right)$. If $H \subseteq H^{\perp}$ we say that $H$ is totally isotropic with respect to $B$. Following the terminology of $\left[\mathbf{T} \mathbf{A}_{\mathbf{2}}\right]$ we call a maximal totally isotropic subgroup a Lagrangian of $B$ on $G$. Clearly a subgroup $L$ of $G$ is a Lagrangian if and only if $L=L^{\perp}$, if and 
only if (by (2.2)) $L$ is totally isotropic and $|L|=\sqrt{|G|}$. Different Lagrangians of $G$ need not be isomorphic, nor even have the same rank. But note that if $L$ is any Lagrangian of $G$, since $G / L \cong L^{\perp}=L$,

$$
\operatorname{rank} L \leq \operatorname{rank} G \leq 2 \operatorname{rank} L .
$$

An extensive analysis of the possible Lagrangians of a group is given in [ $\left.\mathbf{T A}_{\mathbf{3}}\right]$.

It is well known (cf. $\left[\mathbf{T}_{\mathbf{1}}, 1.8\right]$ ) that if $B$ is nondegenerate then $G$ has a symplectic base with respect to $B$, i.e., a base $\left\{g_{1}, h_{1}, g_{2}, h_{2}, \ldots, g_{k}, h_{k}\right\}$ such that for all $i, j$

$$
\begin{aligned}
& B\left(g_{i}, h_{i}\right)=c_{i}, \quad \text { where } \operatorname{ord}\left(g_{i}\right)=\operatorname{ord}\left(h_{i}\right)=\operatorname{ord}\left(c_{i}\right) ; \\
& B\left(g_{i}, g_{j}\right)=B\left(h_{i}, h_{j}\right)=1 \quad \text { and, if } i \neq j, \quad B\left(g_{i}, h_{j}\right)=1 .
\end{aligned}
$$

Such a base can be chosen with $\operatorname{ord}\left(c_{2}\right)\left|\operatorname{ord}\left(c_{1}\right), \operatorname{ord}\left(c_{3}\right)\right| \operatorname{ord}\left(c_{2}\right), \ldots, \operatorname{ord}\left(c_{k}\right) \mid$ $\operatorname{ord}\left(c_{k-1}\right)$, and $\operatorname{ord}\left(c_{k}\right)>1$. Then $\operatorname{ord}\left(c_{1}\right), \operatorname{ord}\left(c_{1}\right), \ldots, \operatorname{ord}\left(c_{k}\right), \operatorname{ord}\left(c_{k}\right)$ are the invariant factors of $G$. The existence of a symplectic base is easy to verify by an orthogonalization process like the one for finding a symplectic base of a symplectic pairing on a vector space. Note that a finite abelian group $G$ admits a nondegenerate symplectic pairing if and only if $G \cong H \times H$ for some group $H$, and this holds if and only if every invariant factor of $G$ occurs an even number of times.

Suppose $G_{1}$ and $G_{2}$ are finite abelian groups with symplectic pairings $B_{i}: G_{i} \times$ $G_{i} \rightarrow C, i=1,2$. Their orthogonal sum $G_{1} \perp G_{2}$ is the direct product $G_{1} \times G_{2}$ together with the symplectic pairing $B:\left(G_{1} \times G_{2}\right) \times\left(G_{1} \times G_{2}\right) \rightarrow C$ given by $B\left(\left(g_{1}, g_{2}\right),\left(h_{1}, h_{2}\right)\right)=B_{1}\left(g_{1}, h_{1}\right) \cdot B_{2}\left(g_{2}, h_{2}\right)$. Clearly $\operatorname{rad}\left(G_{1} \perp G_{2}\right)=\operatorname{rad}\left(G_{1}\right) \times$ $\operatorname{rad}\left(G_{2}\right)$, so $B$ is nondegenerate if and only if $B_{1}$ and $B_{2}$ are both nondegenerate.

Let $A$ be an algebra (with 1 ) over a field $F$, with $\operatorname{dim}_{F} A<\infty$. Identify $F$ with $F \cdot 1 \subseteq A$. For $a \in A^{*}$ let $\tilde{a}$ denote the image $a F^{*}$ of $a$ in $A^{*} / F^{*}$. Let $A$ be a subgroup of $A^{*} / F^{*}$, say $\mathcal{A}=\left\{\tilde{a}_{1}, \tilde{a}_{2}, \ldots\right\}$. Let $F[\mathcal{A}]$ denote the $F$-subspace of $\mathcal{A}$ spanned by $\left\{a_{1}, a_{2}, \ldots\right\}$. Note that $F[A]$ depends only on $A$, independent of the choice of representatives $a_{i}$ for $\tilde{a}_{i} \in \mathcal{A}$. Since $\mathcal{A}$ is a group $F[A]$ is also the subalgebra of $A$ generated by $\left\{a_{1}, a_{2}, \ldots\right\}$.

DEFINITION. For any finite-dimensional $F$-algebra $A$, a subgroup $A$ of $A^{*} / F^{*}$ is an armature of $A$ if $\mathcal{A}$ is abelian, $|\mathcal{A}|=\operatorname{dim}_{F} A$, and $F[A]=A$.

Note that if $\mathcal{A}=\left\{\tilde{a}_{1}, \ldots, \tilde{a}_{n}\right\}$, the last two conditions for an armature are equivalent to: $\left\{a_{1}, \ldots, a_{n}\right\}$ is an $F$-base of $A$. The notion of an armature was introduced in $\left[\mathbf{T}_{\mathbf{1}}, \S 1\right]$. However, the definition given here is different from and slightly nore restrictive than the earlier one, but we allow armatures in algebras other than division rings. Here are some examples of armatures (cf. [ $\mathbf{T}_{\mathbf{1}}$, Example 1.2]).

EXAMPLES 2.4. (a) Let $A$ be a field which is a Kummer extension of $F$, with $[A: F]<\infty$. Let $l=\exp (\mathcal{G}(A / F))$ and let $S=\left\{a \in A^{*} \mid a^{l} \in F\right\}$. Then

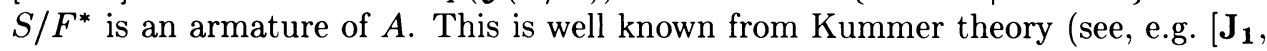
pp. 119-123]). Indeed, $S / F^{*}$ is the only armature of $A$ with exponent dividing $l$.

(b) Let $\omega$ be a primitive $n$th root of unity in $F$. For any $a, b \in F^{*}$, let $A_{\omega}(a, b ; F)$ denote the $F$-algebra with generators: $i, j$, and relations: $i^{n}=a, j^{n}=b, i j=\omega j i$. We will call such an algebra a symbol algebra, terminology suggested by T.-Y. Lam. (Such an algebra is often called a cyclic algebra or a "cyclic" algebra as in [Ta, p. 265]. We prefer to reserve the term cyclic algebra for a crossed-product algebra whose associated Galois field extension is cyclic.) It is well known (cf. [M, $\S 15])$, that $A_{\omega}(a, b ; F)$ is a central simple $F$-algebra of dimension $n^{2}$ over $F$; clearly $\left\{\widetilde{i^{r} j^{s}} \mid 0 \leq r<n, 0 \leq s<n\right\}$ is an armature of $A_{\omega}(a . b ; F)$. 
(c) If $A$ is an armature of an $F$-algebra $A$ and $B$ is any subgroup of $A$, then $B$ is an armature of $F[B]$.

(d) Suppose $A_{1}$ and $A_{2}$ are $F$-algebras with respective armatures $A_{1}$ and $A_{2}$. Then $A:=\left\{(a \otimes b) F^{*} \mid \tilde{a} \in A_{1}\right.$ and $\left.\tilde{b} \in A_{2}\right\}$ is an armature of $A_{1} \otimes_{F} A_{2}$. Clearly $A \cong A_{1} \times A_{2}$.

(e) Suppose $A$ is an armature of an $F$-algebra $A$. Let $K$ be any field containing $F$. Then $A_{K}:=\left\{(a \otimes 1) K^{*} \mid \tilde{a} \in A\right\}$ is an armature of the $K$-algebra $A \otimes_{F} K$.

Let $A$ be an $F$-algebra with armature $A$. As was observed in $\left[\mathbf{T}_{\mathbf{1}}, 1.3\right]$ there is an associated pairing $B_{A}: A \times A \rightarrow F^{*}$ defined by $B_{A}(\tilde{a}, \tilde{b})=[a, b]=a b a^{-1} b^{-1}$. Since $F^{*} \subseteq Z(A), B_{A}(\tilde{a}, \tilde{b})$ is independent of the choice of representatives $a, b$ for $\tilde{a}, \tilde{b}$, and $B_{A}(\tilde{a}, \tilde{b}) \in F^{*}$ as $A$ is abelian. From the general identity $[r, s t]=[r, s] s[r, t] s^{-1}$ it follows that $B_{A}$ is bimultiplicative. Thus, as $A$ is finite, the image of $B_{A}$ is a finite subset of $\mu(F)$. Since $\mu(F)$ embeds in $\mathbf{Q} / \mathbf{Z}, B_{A}$ is a symplectic pairing on $A$.

LEMMA 2.5. Let $A$ be an armature of an $F$-algebra $A$, and let $B$ and $C$ be any subgroups of $A$. Then,

(i) the centralizer in $A$ of $F[B]$ is $F\left[B^{\perp}\right]$;

(ii) $F[B]$ is commutative if and only if $B$ is totally isotropic;

(iii) $Z(A)=F[\operatorname{rad}(A)]$; hence $B_{A}$ is nondegenerate if and only if $Z(A)=F$;

(iv) if $A=B \perp C$, then $A \cong F[B] \otimes_{F} F[C]$.

PROOF. (Of course $B^{\perp}$ and $\operatorname{rad}(A)$ are relative to the symplectic structure on $A$ given by $B_{A}$.) Let $A=\left\{\tilde{a}_{1}, \ldots, \tilde{a}_{n}\right\}$, so $a_{1}, \ldots, a_{n}$ are an $F$-base of $A$. Take any $d \in A$ and write $d=\sum c_{i} a_{i}$ with $c_{i} \in F$. For any $\tilde{b} \in B$, we have

$$
d b-b d=\sum c_{i}\left(a_{i} b-b a_{i}\right)=\sum c_{i}\left(1-B_{\mathcal{A}}\left(\tilde{b}, \tilde{a}_{i}\right)\right) a_{i} b .
$$

Since $b \in A^{*}, a_{1} b, \ldots, a_{n} b$ are $F$-linearly independent. Hence, $d$ commutes with $b$ if and only if for each $i, c_{i}=0$ or $B_{A}\left(\tilde{b}, \tilde{a}_{i}\right)=1$. Thus $d$ centralizes $F[B]$ if and only if $d$ commutes with every $b$ with $\tilde{b} \in B$, if and only if $c_{i}=0$ whenever $\tilde{a}_{i} \notin B^{\perp}$, if and only if $d \in F\left[B^{\perp}\right]$. This proves (i), and (ii) and (iii) follow immediately.

For (iv), suppose $A=B \perp C$. By this we mean that $A$ is the direct product of its subgroups $B$ and $C$, and $C \subseteq B^{\perp}$. So $F[C]$ centralizes $F[B]$, and the inclusions $F[B] \hookrightarrow A, F[C] \hookrightarrow A$ induce an $F$-algebra homomorphism $\alpha: F[B] \otimes_{F} F[C] \rightarrow A$. This map is surjective since $B$ and $C$ generate $A$, which lifts to an $F$-base of $A$. By dimension count, $\alpha$ is an isomorphism.

REMARK 2.6. Let $A$ be an armature of an $F$-algebra $A$. Then $A$ yields an explicit presentation of $A$ as follows: Let $\tilde{a}_{1}, \ldots, \tilde{a}_{k}$ be a base of the abelian group $\AA$, let $n_{i}=\operatorname{ord}\left(\tilde{a}_{i}\right)$, and let $c_{i}=a_{i}^{n_{i}} \in F^{*} .\left(c_{i} \in F^{*}\right.$ as $\tilde{a}_{i}^{n_{i}}=1 \AA$. The coset $c_{i} F^{* n_{i}}$ is uniquely determined by $\tilde{a}_{i}$, though $c_{i}$ depends on the choice of representative $a_{i}$ of $\tilde{a}_{i}$.) Then $A$ is isomorphic to the $F$-algebra with generators: $z_{1}, \ldots, z_{k}$ and relations: $z_{i}^{n_{i}}=c_{i}, z_{i} z_{j}=B_{\mathcal{A}}\left(\tilde{a}_{i}, \tilde{a}_{j}\right) z_{j} z_{i}$. For, if $A^{\prime}$ is the algebra with these generators and relations, then there is a surjective homomorphism $A^{\prime} \rightarrow A$ given by $z_{i} \mapsto a_{i}$. This map is an isomorphism, since it is easy to check that $\operatorname{dim}_{F} A^{\prime} \leq$ $n_{1} \cdots n_{k}=|A|=\operatorname{dim}_{F} A$.

Proposition 2.7. Let $A$ be a finite-dimensional $F$-algebra with $Z(A)=F$. Then the following statements are equivalent.

(i) $A$ has an armature.

(ii) $A$ is isomorphic to a tensor product of symbol algebras over $F$. 
ProOF. (ii) $\Rightarrow$ (i) is immediate from Examples 2.4(b) and (d).

(i) $\Rightarrow$ (ii) Let $A$ be an armature on $A$, and $B_{\mathcal{A}}$ the associated symplectic pairing. Since $Z(A)=F$ the lemma says that $B_{A}$ is nondegenerate. So, $A$ has a symplectic base $\left\{\tilde{a}_{1}, \tilde{b}_{1}, \ldots, \tilde{a}_{k}, \tilde{b}_{k}\right\}$. Let $\omega_{i}=B_{\mathcal{A}}\left(\tilde{a}_{i}, \tilde{b}_{i}\right) \in \mu(F)$, and let $n_{i}=$ $\operatorname{ord}\left(\omega_{i}\right)=\operatorname{ord}\left(\tilde{a}_{i}\right)=\operatorname{ord}\left(\tilde{b}_{i}\right)$. Let $c_{i}=a_{i}^{n_{i}} \in F^{*}$ and $d_{i}=b_{i}^{n_{i}} \in F^{*}$, and let $A_{i}$ be the subgroup of $A$ generated by $\tilde{a}_{i}, \tilde{b}_{i}$. Then the presentation given above shows that $F\left[A_{i}\right] \cong A_{\omega_{i}}\left(c_{i}, d_{i} ; F\right)$. Since $A=A_{1} \perp \cdots \perp A_{k}$ the lemma says $A \cong \bigotimes_{i=1}^{k} A_{\omega_{i}}\left(c_{i}, d_{i} ; F\right)$.

COROLlaRY 2.8 (cf. [ $\mathbf{T}_{\mathbf{1}}$, Proposition 1.9]). If $A$ is an $F$-algebra with armature $A$ and $Z(A)$ is a field, then $A$ is isomorphic to a tensor product of symbol algebras over $Z(A)$.

Proof. Let $K=Z(A)=F[\operatorname{rad}(A)]$. Let $A^{\prime}$ be the image of $A$ in the natural homomorphism $g: A^{*} / F^{*} \rightarrow A^{*} / K^{*}$. Then $K\left[A^{\prime}\right]=F[A]=A$. Since $|\operatorname{rad}(\mathcal{A})|=$ $[K: F]$ and the inverse images in $A$ of elements of $A$ are $F$-linearly independent, we have $\operatorname{ker}(g) \cap A=K^{*} \cap A=\operatorname{rad}(A)$. Hence,

$$
\left|\mathcal{A}^{\prime}\right|=|\mathcal{A}| /|\operatorname{rad}(\mathcal{A})|=\left(\operatorname{dim}_{F} A\right) /[K: F]=\operatorname{dim}_{K} A .
$$

Therefore, $A^{\prime}$ is an armature of $A$ as a $K$-algebra, and Proposition 2.7 yields the corollary.

Now, suppose $A$ is a commutative $F$-algebra with armature $A$, and let $l$ be a positive integer with $\exp (A) \mid l$. Then there is a well-defined group homomorphism $f: A \rightarrow F^{*} / F^{* l}$ given by $\tilde{a} \mapsto a^{l} F^{* l}$. Let $\tilde{a}_{1}, \ldots, \tilde{a}_{k}$ be a base of $\mathcal{A}$, and let $\operatorname{ord}\left(\tilde{a}_{i}\right)=n_{i}$ and $a_{i}^{n_{i}}=c_{i} \in F^{*}$. In the extreme cases for $f$ we obtain a nice description of $A$.

LEMMA 2.9. Suppose $\mu_{l} \subseteq F$. Then,

(a) if $f$ is injective, then $A \cong F\left(c_{1}^{1 / n_{1}}, \ldots, c_{k}^{1 / n_{k}}\right)$, a field;

(b) if $\operatorname{ker}(f)=A$, then $A \cong F \oplus F \oplus \cdots \oplus F$.

Proof. (a) From the presentation of $A$ in (2.6) there is clearly a surjective $F$-algebra homomorphism $h: A \rightarrow F\left(c_{1}^{1 / n_{1}}, \ldots, c_{k}^{1 / n_{k}}\right)$ given by $a_{i} \mapsto c_{i}^{1 / n_{i}}$. By Kummer theory $\left[F\left(c_{1}^{1 / n_{1}}, \ldots, c_{k}^{1 / n_{k}}\right): F\right]$ equals the order of the subgroup of $F^{*} / F^{* l}$ generated by $\left\{c_{1}^{l / n_{1}}, \ldots, c_{k}^{l / n_{k}}\right\}$. This group is $\operatorname{im}(f)$, so assuming $f$ is injective, its order is $|A|=\operatorname{dim}_{F} A$. Thus a dimension comparison shows $h$ is an isomorphism.

(b) Suppose $\operatorname{ker}(f)=A$. Then, for each $i, c_{i}^{l / n_{i}}=a_{i}^{l} \in F^{* l}$. Say $c_{i}^{l / n_{i}}=d_{i}^{l}$, for some $d_{i} \in F^{*}$. Then $c_{i}=\omega_{i} d_{i}^{n_{i}}$ for some $\omega_{i} \in \mu_{l / n_{i}}(F)$. Since $\mu_{l} \subseteq F, \omega_{i}$ has an $n_{i}$ th root in $F$, and we can write $c_{i}=e_{i}^{n_{i}}$ for some $e_{i} \in F^{*}$. From the presentation given in (2.6) for $A$ we have $A \cong F\left[z_{1}, \ldots, z_{k}\right] /\left(z_{1}^{n_{1}}-c_{1}, \ldots, z_{k}^{n_{k}}-c_{k}\right)$, where the $z_{i}$ are commuting indeterminates. Since each $z_{i}^{n_{i}}-c_{i}=z_{i}^{n_{i}}-e_{i}^{n_{i}}$ factors into $n_{i}$ distinct linear factors, $A$ is a direct sum of copies of $F$.

3. Totally ramified tame division algebras. Let $(D, v)$ be any valued division algebra which is tame and totally ramified over its center $F$. (Recall that this means: $\left|\Gamma_{D}: \Gamma_{F}\right|=[D: F]<\infty$, so $\bar{D}=\bar{F}$, and $\operatorname{char}(\bar{F}) \nmid[D: F]$.) The finite abelian group $\Gamma_{D} / \Gamma_{F}$ is an important invariant of $D$, which we call the relative 
value group of $D$ with respect to $v$. We will define a natural pairing on $\Gamma_{D} / \Gamma_{F}$ via commutators, and will show that this pairing carries substantial information about the structure of $D$.

Define a function $g: D^{*} \times D^{*} \rightarrow \bar{F}^{*}$ by

$$
g(d, e)=\overline{\operatorname{ded}^{-1} e^{-1}} \text {. }
$$

This function is well-defined as $\Gamma_{D}$ is abelian, so all commutators lie in $U_{D}$. Since any inner automorphism of $D$ maps the valuation ring $V_{D}$ to itself and induces the trivial automorphism on $\bar{D}=\bar{F}$, we have

$$
\begin{aligned}
g\left(d, e_{1} e_{2}\right) & =\overline{d e_{1} e_{2} d^{-1} e_{2}^{-1} e_{1}^{-1}}=\overline{d e_{1} d^{-1} e_{1}^{-1}} \cdot \overline{e_{1}\left(d e_{2} d^{-1} e_{2}^{-1}\right) e_{1}^{-1}} \\
& =g\left(d, e_{1}\right) g\left(d, e_{2}\right) .
\end{aligned}
$$

Thus, $g$ is multiplicative in the second component, likewise in the first component, and is clearly symplectic. Note that the radical of $g$ contains $F^{*}$ and the unit group $U_{D}$. Because $D^{*} / F^{*} U_{D} \cong \Gamma_{D} / \Gamma_{F}, g$ induces a (bilinear) symplectic pairing

$$
C_{D}:\left(\Gamma_{D} / \Gamma_{F}\right) \times\left(\Gamma_{D} / \Gamma_{F}\right) \rightarrow \bar{F}^{*},
$$

which we call the canonical pairing of $v$ on $D$.

PROPOSITION 3.1. For any valued division algebra $(D, v)$ tame and totally ramified over its center $F$, the canonical pairing $C_{D}$ on $\Gamma_{D} / \Gamma_{F}$ is nondegenerate. Consequently, $\mu_{l} \subseteq \bar{F}$, where $l=\exp \left(\Gamma_{D} / \Gamma_{F}\right)$, and $\Gamma_{D} / \Gamma_{F} \cong H \times H$ for some finite abelian group $H$.

ProOF. Assume first that $(F, v)$ is Henselian and $\mu_{l} \subseteq F$, so $\mu_{l} \subseteq \bar{F}$. Take any $a \in D^{*}$ with $v(a) \notin \Gamma_{F}$. We show that $\overline{v(a)}:=v(a)+\Gamma_{F}$ is not in $\operatorname{rad}\left(\Gamma_{D} / \Gamma_{F}\right)$. Let $n=\operatorname{ord}(\overline{v(a)})$ in $\Gamma_{D} / \Gamma_{F}$, so $n \mid l$. Then there is a $b \in F^{*}$ with $v(b)=v\left(a^{n}\right)$ and, as $\bar{D}=\bar{F}$, a $c \in U_{F}$ with $\overline{a^{n} b^{-1}}=\bar{c}$ in $\bar{D}$. So $\overline{a^{n} b^{-1} c^{-1}}=1$ in $\overline{F(a)}$. Since $F(a)$ is Henselian and $\mu_{n} \subseteq F(a)$ every 1-unit of $F(a)$ has an $n$th root in $F(a)$; so there is a $u \in F(a)$ with $v(u)=0$ and $u^{n}=a^{n} b^{-1} c^{-1}$. Let $a^{\prime}=a u^{-1}$. Then $a^{\prime n}=b c \in F^{*}$ with $v\left(a^{\prime}\right)=v(a)$. Also $\left[F\left(a^{\prime}\right): F\right] \geq\left|\Gamma_{F\left(a^{\prime}\right)}: \Gamma_{F}\right| \geq$ ord $\overline{v\left(a^{\prime}\right)}=n$. Hence, by Kummer theory, for any primitive $n$th root of unity $\omega \in F^{*}$ there is an $F$-automorphism $\tau$ of $F\left(a^{\prime}\right)$ with $\tau\left(a^{\prime}\right)=a^{\prime} \omega$. The Skolem-Noether theorem shows there is a $d \in D^{*}$ with $d a^{\prime} d^{-1}=\tau\left(a^{\prime}\right)=a^{\prime} \omega$. Then $C_{D}\left(\overline{v(d)}, \overline{v\left(a^{\prime}\right)}\right)=$ $\overline{d a^{\prime} d^{-1} a^{\prime-1}}=\bar{\omega}$. Because $\operatorname{char}(\bar{F}) \nmid n$, ord $(\bar{\omega})=\operatorname{ord}(\omega)=n$, so $\bar{\omega} \neq 1$. Thus, $\overline{v(a)}=\overline{v\left(a^{\prime}\right)} \notin \operatorname{rad}\left(\Gamma_{D} / \Gamma_{F}\right)$, proving the nondegeneracy of $C_{D}$.

Now drop the restrictions on $F$. Let $\left(F_{h}, v\right)$ be a Henselization of $(F, v)$ and let $K=F_{h}(\lambda)$ where $\lambda$ is a primitive $l$ th root of unity. Because $F_{h}$ is Henselian, $v$ extends uniquely to $K$; further, $K$ is Henselian and an inertial extension of $F_{h}$, which is an immediate extension of $F$. Hence, $\Gamma_{K}=\Gamma_{F_{h}}=\Gamma_{F}$. By the extension Lemma 1.6, $D \otimes_{F} K$ is a division algebra totally ramified over $K$, and $\Gamma_{D \otimes K}=\Gamma_{D}$. When we identify $\Gamma_{D} / \Gamma_{F}$ with $\Gamma_{D \otimes K} / \Gamma_{K}$, the canonical pairing for $D$ is the same as the pairing for $D \otimes_{F} K$, which we have seen to be nondegenerate. The nondegeneracy assures that the image of $C_{D}$ contains all $l$ th roots of unity and, as noted in $\S 2$, that $\Gamma_{D} / \Gamma_{F}$ has the form $H \times H$.

The fact that $\mu_{l} \subseteq \bar{F}$ was noted in the Henselian case in [PY, Proposition 5].

REMARKS 3.2. (a) If $D$ is a division algebra with valuation tame and totally ramified over its center $F$. then the nondegenerate pairing assures that $\Gamma_{D} / \Gamma_{F}$ has 
even rank as an abelian group. Since $\operatorname{dim}_{\mathbf{Q}}\left(\Gamma_{F} \otimes_{\mathbf{z}} Q\right) \geq \operatorname{rank}\left(\Gamma_{D} / \Gamma_{F}\right) \geq 2$ if $D \neq F$, we must have $\Gamma_{F} \neq \mathbf{Z}$. Thus, discrete (rank 1) valued fields do not admit totally ramified division algebras. This fact may explain why totally ramified division algebras were not studied many years earlier.

(b) In order to define the canonical pairing on $D$, it suffices to have a valuation on $D$ with $\bar{D}=\overline{Z(D)}$. This does not lead to a generalization of Proposition 3.1, however, since it can be shown that if $D$ is tame over $Z(D)$ and $\bar{D}=\overline{Z(D)}$, then $D$ is totally ramified over $Z(D)$.

The next proposition generalizes a result of the first author $\left[\mathbf{T}_{\mathbf{1}}\right.$, Theorem 3.6] on the possible cyclic decompositions of certain twisted iterated Laurent series division algebras. Note that its proof uses the existence of the canonical pairing, but not its nondegeneracy.

Proposition 3.3. Let $(D, v)$ be a valued division algebra with $D$ tame and totally ramified over $Z(D)=F$. If $A$ is an armature of $D$ as an $F$-algebra then the map $\bar{v}: A \rightarrow \Gamma_{D} / \Gamma_{F}$ induced by $v$ is an isomorphism. Consequently, if $D \cong$ $A_{1} \otimes_{F} \cdots \otimes_{F} A_{k}$, where the $A_{i}$ are symbol algebras with $\operatorname{dim}_{F} A_{i}=n_{i}^{2}>1$ and $n_{2}\left|n_{1}, n_{3}\right| n_{2}, \ldots, n_{k} \mid n_{k-1}$, then $n_{1}, n_{1}, n_{2}, n_{2}, \ldots, n_{k}, n_{k}$ are uniquely determined as the invariant factors of $\Gamma_{D} / \Gamma_{F}$.

ProOF. The armature pairing $B_{\mathcal{A}}$ on $\mathcal{A}$ (see $\S 2$ ) and the canonical pairing $C_{D}$ are both based on commutators. Thus, it is easy to see that the following diagram is commutative:

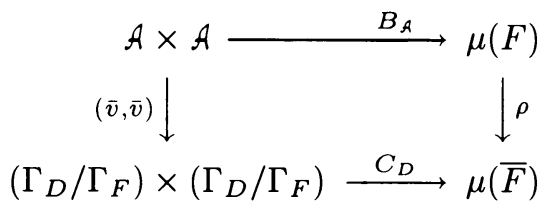

where $\rho: V_{F} \rightarrow \bar{F}$ is the natural projection. (This diagram is analogous to (1.5) for field extensions.) Because $B_{\AA}$ is nondegenerate by (2.5)(iii) and $\rho$ is injective on roots of unity of order prime to $\operatorname{char}(\bar{F}), \bar{v}$ must be injective. Hence $\bar{v}$ is an isomorphism, as $|A|=[D: F]=\left|\Gamma_{D}: \Gamma_{F}\right|$. If $D$ is a tensor product of symbol algebras of dimensions $n_{i}^{2}$, we saw in $\S 2$ that $D$ has an armature $A$ with $A \cong \prod_{i=1}^{k}\left(\mathbf{Z} / n_{i} \mathbf{Z}\right) \times\left(\mathbf{Z} / n_{i} \mathbf{Z}\right)$. The rest of the corollary follows immediately from this.

Given a commutative diagram such as (3.4) (with a canonical isomorphism between $\operatorname{im}\left(B_{A}\right)$ and $\left.\operatorname{im}\left(C_{D}\right)\right)$ we say that the isomorphism $\bar{v}$ is an isometry between the nondegenerate pairings $B_{\AA}$ and $C_{D}$.

We recall from $[\mathbf{J W}]$ a useful device for constructing totally ramified division algebras. Let $A$ be a central simple $F$-algebra, where $F$ is a field with valuation $v$. Let $\Delta$ be the divisible hull of $\Gamma_{F}$. Suppose $A$ has an armature $A$. Then there is a well-defined function $\bar{w}: A \rightarrow \Delta / \Gamma_{F}$ given by $a F^{*} \mapsto(1 / n) v\left(a^{n}\right)+\Gamma_{F}$, where $n=|A|=\operatorname{dim}_{F} A$.

PROPOSITION 3.5. The map $\bar{w}$ just defined is a group homomorphism. Suppose $\bar{w}$ is injective. Then $A$ is a division algebra and $v$ extends to a valuation on $A$ with $A$ totally ramified over $F ; \Gamma_{A}$ is the subgroup of $\Delta$ such that $\Gamma_{A} / \Gamma_{F}=\operatorname{im}(\bar{w})$; and $\bar{w}=\bar{v}$ (where $\bar{v}$ is the map in Proposition 3.3). 
ProOF. This is a special case of [JW, Theorem 2.5], with $L=F$. The valuation on $A$ is defined by taking inverse images $a_{1}, \ldots, a_{n} \in A^{*}$ of the elements $a_{i} F^{*}$ of $A$ and setting

$$
v\left(\sum c_{i} a_{i}\right)=\inf \left\{\frac{1}{n} v\left(a_{i}^{n}\right)+v\left(c_{i}\right) \mid c_{i} \neq 0\right\}
$$

for any $c_{i} \in F$ not all zero.

If $(F, v)$ is Henselian and $\bar{F}$ is separably closed, we will describe the structure of $A$ even when $\bar{w}$ is not injective-see Theorem 4.3 below.

EXAMPLES 3.6. (a) Let $n_{1}, \ldots, n_{k}$ be any positive integers, and let $F_{0}$ be any field containing primitive $n_{i}$ th roots of unity $\omega_{i}, i=1,2, \ldots, k$. Let $x_{1}, \ldots, x_{2 k}$ be $2 k$ independent indeterminantes over $F_{0}$, and let $F=F_{0}\left(x_{1}, \ldots, x_{2 k}\right)$. Let $F^{\prime}=F_{0}\left(\left(x_{1}\right)\right) \cdots\left(\left(x_{2 k}\right)\right)$ be the iterated Laurent power series field; that is, $F^{\prime}=F_{2 k}$ where for each $m, F_{m}=F_{m-1}\left(\left(x_{m}\right)\right):=\left\{\sum_{l=n}^{\infty} c_{l} x_{m}^{l} \mid n \in \mathbf{Z}\right.$ and all $\left.c_{l} \in F_{m-1}\right\}$. There is a standard valuation $v: F^{* *} \rightarrow \mathbf{Z}^{2 k}$ given by

$$
v\left(\sum_{l_{1}} \cdots \sum_{l_{2 k}} c_{l_{1} \cdots l_{2 k}} x_{1}^{l_{1}} \cdots x_{2 k}^{l_{2 k}}\right)=\inf \left\{\left(l_{1}, \ldots, l_{2 k}\right) \mid c_{l_{1} \cdots l_{2 k}} \neq 0\right\},
$$

where $\mathbf{Z}^{2 k}$ is given the right-to-left lexicographical ordering. In this ordering $\left(l_{1}, \ldots, l_{2 k}\right)<\left(m_{1}, \ldots, m_{2 k}\right)$ just when there is a $q$ with $l_{q}<m_{q}$ and $l_{r}=m_{r}$ for $q<r \leq 2 k$. With respect to $v$ we have $\Gamma_{F^{\prime}}=\mathbf{Z}^{2 k}$ and $\overline{F^{\prime}}=F_{0}$, and, as is well known (cf. [Ri, p. 77, Proposition 4; p. 198, Theorem 4]), $F^{\prime}$ is Henselian. This valuation restricts to a valuation on $F$, and $\left(F^{\prime}, v\right)$ is clearly an immediate extension of $(F, v)$.

Now, let

$$
D=\bigotimes_{m=1}^{k} A_{\omega_{m}}\left(x_{2 m-1}, x_{2 m} ; F\right)
$$

and let $i_{m}, j_{m} \in D^{*}$ be generators of $A_{\omega_{m}}\left(x_{2 m-1}, x_{2 m} ; F\right)$ with $i_{m}^{n_{m}}=x_{2 m-1}$, $j_{m}^{n_{m}}=x_{2 m}, i_{m} j_{m}=\omega_{m} j_{m} i_{m}$. Then the subgroup $A$ of $D^{*} / F^{*}$ generated by the $\tilde{\imath}_{m}, \tilde{\jmath}_{m}, 1 \leq m \leq k$, is an armature of $D$, and $\left\{\tilde{\imath}_{1}, \tilde{\jmath}_{1}, \ldots, \tilde{\imath}_{m}, \tilde{\jmath}_{m}\right\}$ is a symplectic base of $A$ with respect to the armature pairing $B_{A}$. Here $\Delta=\mathbf{Q}^{2 k}$ and the map $\bar{w}$ of Proposition 3.5 sends

$$
i_{1} F^{*} \mapsto\left(1 / n_{1}, 0, \ldots, 0\right)+\Gamma_{F}, \ldots, j_{k} F^{*} \mapsto\left(0, \ldots, 0,1 / n_{k}\right)+\Gamma_{F} .
$$

Thus $|\bar{w}(A)| \geq|A|$, which assures that $\bar{w}$ is injective. Hence, the proposition shows that $D$ is a division algebra and $v$ extends to $D$ so that $D$ is totally ramified over $F$. We have

$$
\Gamma_{D}=\prod_{m=1}^{k}\left(\mathbf{Z} / n_{m}\right) \times\left(\mathbf{Z} / n_{m}\right), \quad \text { so } \Gamma_{D} / \Gamma_{F} \cong \prod_{m=1}^{k}\left(\mathbf{Z} / n_{m} \mathbf{Z}\right) \times\left(\mathbf{Z} / n_{m} \mathbf{Z}\right) \text {. }
$$

Likewise, if $L$ is any field, $F \subseteq L \subseteq F^{\prime}$, then $D \otimes_{F} L$ has a valuation extending $v$ on $L$ with $\Gamma_{D \otimes L}=\Gamma_{D}$, and $D \otimes L$ totally ramified over $L$. This can be seen in the same way as for $D$ since $\Gamma_{L}=\Gamma_{F}$, or by using the extension Lemma 1.6. The division algebras of the type $D \otimes_{F} F^{\prime}$ were used by Amitsur in his construction of noncrossed products; the relative value group $\Gamma_{D \otimes F^{\prime}} / \Gamma_{F^{\prime}}$ gives strong control over the possible Galois groups of maximal subfields of $D \otimes F^{\prime}$ (see the comments in [ JW. (2.3) . (2.8)]). Other information about the structure of $D$ determined by $\Gamma_{D} / \Gamma_{F}$ is collected in (4.7) below. 
(b) To be more specific, suppose $F_{0}$ contains a primitive 4 th root of unity $\omega$, and let $k=n_{1}=n_{2}=2$. So, $D$ is the tensor product of two quaternion algebras: $D=\left(\left(x_{1}, x_{2}\right) / F\right) \otimes_{F}\left(\left(x_{3}, x_{4}\right) / F\right)$, where $F=F_{0}\left(x_{1}, \ldots, x_{4}\right)$. It is immediate from Proposition 3.3 that $D$ is not a symbol algebra, i.e., $D$ has no maximal subfield cyclic over $F$. For, any armature $A$ of $D$ must have $A \cong \Gamma_{D} / \Gamma_{F} \cong(\mathbf{Z} / 2 \mathbf{Z})^{4}$, but if $D$ were a symbol algebra, it would have an armature of rank 2 . We will show later (see (4.7)) that $D$ is not split by any cyclic Galois extension of $F$. Draxl shows in $\left[\mathbf{D}_{1}\right.$, pp. 168-169] that for the corresponding power series algebra $D^{\prime}$ of $D\left(D^{\prime}=D \otimes_{F} F^{\prime}\right), S K_{1}\left(D^{\prime}\right) \neq 1$; that is, there is an $a \in D^{* *}$ with $\operatorname{Nrd}(a)=1$ (where Nrd is the reduced norm) but $a$ is not in the commutator group $\left[D^{\prime *}, D^{\prime *}\right]$. This can be seen very easily for $D^{\prime}$ and also for $D$ using the canonical pairing: We take $a=\omega \in F_{0}$. Then, as $[D: F]=4^{2}, \operatorname{Nrd}(\omega)=\omega^{4}=1$. But the construction of the canonical pairing shows that the image of $\left[D^{*}, D^{*}\right]$ in $\bar{D}$ lies in $\mu_{l}(\bar{F})$, where $l=\exp \left(\Gamma_{D} / \Gamma_{F}\right)=2$. Since $\bar{\omega}$ is a primitive 4 th root of unity in $\bar{F}, \omega$ cannot lie in $\left[D^{*}, D^{*}\right]$. Note that the nondegeneracy of the canonical pairing was not needed for this argument. This is a remarkably easy example, considering that it was an open question (the "Tannaka-Artin problem") for some decades whether any finitedimensional division algebra $D$ existed with $S K_{1}(D) \neq 1$. (For more on $S K_{1}$ of division algebras see $[\mathbf{D K}]$ and $[\mathbf{P}]$.)

When conditions are imposed on the valuation on the center, more can be said about the structure of a valued division algebra $D$ that is tame and totally ramified over $Z(D)$. We consider here the case where $(Z(D), v)$ is Henselian, and in $\S 4$ the case where $(Z(D), v)$ is Henselian and $\overline{Z(D)}$ is separably closed. The following theorem of Draxl $\left[\mathbf{D}_{\mathbf{2}}\right.$, Theorem 1] makes the Henselian case quite tractable.

DECOMPOSITION THEOREM 3.7 (DRAXL). Let $(D, v)$ be a valued division algebra which is tame and totally ramified over $F=Z(D)$. If $(F, v)$ is Henselian, then $D$ is isomorpic to a tensor product of symbol algebras.

It is a nice observation of D. Saltman (unpublished) that if the absolute Galois group of a field $K$ is abelian, then every finite-dimensional $K$-central division algebra is isomorphic to a tensor product of symbol algebras. The "local" version of this observation says that if $D$ is an $F$-central division algebra over any field $F$ and if every subfield $L$ of $D$ with $L \supseteq F$ is a Kummer extension of $F$, then $D$ is isomorphic to a tensor product of symbol algebras. Note that Draxl's decomposition theorem is an instance of the local Saltman result. For, with $D$ and $F$ as in (3.7) we have seen in (3.1) that $\mu_{l} \subseteq \bar{F}$; then (1.4)(iii) yields the Kummer condition on the intermediate fields.

For certain Malcev-Neumann division algebras tame and totally ramified over their (Henselian valued) centers, the first author and Amitsur gave in $\left[\mathbf{T A}_{\mathbf{2}}\right.$, Theorem 4.2] a full classification of the subfields of the division algebras, which we now generalize:

THEOREM 3.8. Let $(D, v)$ be a valued division algebra tame and totally ramified over $Z(D)=F$. Assume $F$ is Henselian with respect to $v$. Then there is a 1-1 correspondence between the isomorphism classes of $F$-subalgebras $A$ of $D$ and the subgroups $\Gamma_{A} / \Gamma_{F}$ of $\Gamma_{D} / \Gamma_{F}$. In this correspondence the centralizer of an algebra $A$ corresponds to $\left(\Gamma_{A} / \Gamma_{F}\right)^{\perp}$ relative to the canonical pairing $C_{D}$. Hence, $A$ is a field if and only if $\Gamma_{A} / \Gamma_{F}$ is totally isotropic. 
Proof. As noted above, we have $\mu_{l} \subseteq F$, where $l=\exp \left(\Gamma_{D} / \Gamma_{F}\right)$. By Draxl's Theorem 3.7 and by $(2.4)(\mathrm{b})$ and (d) above $D$ has an armature $D$, say $D=\left\{\tilde{d}_{1}, \ldots, \tilde{d}_{n}\right\}$, where $\tilde{d}_{i}=d_{i} F^{*}$. We have seen that the map of $(3.3) \bar{v}: D \rightarrow$ $\Gamma_{D} / \Gamma_{F}$ is an isomorphism. For any $F$-subalgebra $A$ of $D$, let $B=\bar{v}^{-1}\left(\Gamma_{A} / \Gamma_{F}\right) \subseteq D$. Observe that $\Gamma_{F[B]}=\Gamma_{A}$. We will show that $A \cong F[B]$.

First, take any $a \in A^{*}$, and suppose $a^{r} \in F^{*}$, where $r=\operatorname{ord}\left(v(a)+\Gamma_{F}\right)$ in $\Gamma_{D} / \Gamma_{F}$. There is a unique $\tilde{d}_{j} \in D$ with $\bar{v}\left(\tilde{d}_{j}\right)=v(a)+\Gamma_{F}$. Then $\tilde{d}_{j} \in B$, and we claim that $a^{r}=s^{r} d_{j}^{r}$ for some $s \in F^{*}$. To see this, first note that since $\bar{D}=\bar{F}$ and $v(a) \equiv v\left(d_{j}\right)\left(\bmod \Gamma_{F}\right)$ there exist $e \in F^{*}$ and $m \in D^{*}$ with $v(m)>0$ such that $d_{j}=a e(1+m)$. Because ord $\left(\tilde{d}_{j}\right)($ in $D)=\operatorname{ord}\left(\bar{v}\left(\tilde{d}_{j}\right)\right)\left(\right.$ in $\left.\Gamma_{D} / \Gamma_{F}\right)=r, d_{j}^{r} \in F^{*}$. But

$$
d_{j}^{r}=e^{r} a(1+m) a^{-1} a^{2}(1+m) a^{-2} \cdots a^{r}(1+m) a^{-r} \cdot a^{r} .
$$

Since $a^{k}(1+m) a^{-k}=1+a^{k} m a^{-k} \in 1+M_{D}$ for every $k$, we have $d_{j}^{r} e^{-r} a^{-r} \in$ $\left(1+M_{D}\right) \cap F=1+M_{F}$. The claim then follows, since $1+M_{F} \subseteq F^{* r}$ as $F$ is Henselian and $\operatorname{char}(\bar{F}) \nmid r$.

In verifying $A \cong F[B]$, we first consider some special cases. Suppose $A$ is a field. Because $A$ is commutative $\Gamma_{A} / \Gamma_{F}$ is totally isotropic with respect to $C_{D}$. Hence (cf. (3.3)) $B$ must be totally isotropic with respect to the armature pairing $B_{D}$; so $F[B]$ is commutative. Since $A$ is totally ramified over $F$, which is Henselian and contains enough roots of unity, $A$ is a Kummer extension of $F$. Hence, $A$ has an armature $A=\left\{a \in A^{*} \mid a^{l} \in F^{*}\right\} \subseteq A^{*} / F^{*}$, where $l=\exp (\mathcal{G}(A / F))$. As noted in $\S 1, A$ maps isomorphically to $\Gamma_{A} / \Gamma_{F}$. Since also $B \cong \Gamma_{A} / \Gamma_{F}$, these isomorphisms combine to give an isomorphism $g: A \rightarrow B$. Take a base $\left\{\tilde{a}_{1}, \ldots, \tilde{a}_{k}\right\}$ of the abelian group $A$, and let $\tilde{b}_{i}=g\left(\tilde{a}_{i}\right)$. The claim proved above shows that we can choose representatives $a_{i}$ of $\tilde{a}_{i}$ and $b_{i}$ of $\tilde{b}_{i}$ so that $a_{i}^{r_{i}}=b_{i}^{r_{i}} \in F$, where $r_{i}=\operatorname{ord}\left(\tilde{a}_{i}\right)=\operatorname{ord}\left(\tilde{b}_{i}\right)$. Hence, by the presentation given in (2.6), $A=F[\mathcal{A}] \cong$ $F\left[z_{1}, \ldots, z_{k}\right] /\left(z_{1}^{r_{1}}-a_{1}^{r_{1}}, \ldots, z_{k}^{r_{k}}-a_{k}^{r_{k}}\right) \cong F[B]$, as desired.

Instead of assuming $A$ is a field, now assume that $Z(A)=F$. By Draxl's decomposition Theorem 3.7, $A$ has an armature as an $F$-algebra, which again we call $A$. From the isomorphism $A \rightarrow \Gamma_{A} / \Gamma_{F}$ (see (3.3)) we obtain as before an isomorphism $g: A \rightarrow B$. Let $B_{A}$ and $B_{B}$ be the armature pairings for $A$ and $B$. For any $\tilde{a}, \tilde{c} \in \mathcal{A}, B_{\mathcal{A}}(\tilde{a}, \tilde{c})$ is a root of unity whose image in $\bar{F}$ is determined by the images of $\tilde{a}$ and $\tilde{c}$ in $\Gamma_{D} / \Gamma_{F}$. Therefore the map $g$ is an isometry between $B_{\mathcal{A}}$ and $B_{B}$. Choose a base $\left\{\tilde{a}_{1}, \ldots, \tilde{a}_{k}\right\}$ of $\mathcal{A}$. As in the field case we can choose representatives $a_{i}$ of $\tilde{a}_{i}$ in $A$ and $b_{i}$ of $g\left(\tilde{a}_{i}\right)$ in $F[B]$ so that $a_{i}^{r_{i}}=b_{i}^{r_{i}}$, where $r_{i}=\operatorname{ord}\left(\tilde{a}_{i}\right)=\operatorname{ord}\left(g\left(\tilde{a}_{i}\right)\right)$. Then the presentation (2.6) of $F[A]$ using the $a_{i}$ is the same as the presentation of $F[B]$ using the $b_{i}$. Thus, $A=F[A] \cong F[B]$.

Finally, for the general case we drop the restrictions on $A$. Let $E=Z(A)$, and let $\mathcal{E}=\bar{v}^{-1}\left(\Gamma_{E} / \Gamma_{F}\right) \subseteq \mathcal{A}$. By the field case $E \cong F|\mathcal{E}|$. By Skolem-Noether, we may, after conjugating $A$ by an inner automorphism, assume $E=F[\mathcal{E}]$. Let $\mathcal{E}^{\perp}$ be the orthogonal dual of $\mathcal{E}$ with respect to $B_{\mathcal{D}}$, and let $D^{\prime}=F\left[\mathcal{E}^{\perp}\right]$, which by $(2.5)$ is the centralizer of $E$ in $D$. Observe that $\mathcal{E} \subseteq B \subseteq \mathcal{E}^{\perp}$; the latter inclusion holds because $B_{D}$ is isometric to $C_{D}$, and $C_{D}\left(\Gamma_{F[B]} / \Gamma_{F}, \Gamma_{E} / \Gamma_{F}\right)=C_{D}\left(\Gamma_{A} / \Gamma_{F}, \Gamma_{E} / \Gamma_{F}\right)=1$. Let $h: \mathcal{E}^{\perp} \rightarrow D^{\prime *} / E^{*}$ be the map given by $c F^{*} \mapsto c E^{*}$. Note that $\operatorname{ker}(h)=\mathcal{E}$ since $\left(E^{*} / F^{*}\right) \cap D=\mathcal{E}$. Therefore, as $E\left[h\left(\mathcal{E}^{\perp}\right)\right]=F\left[\mathcal{E}^{\perp}\right]=D^{\prime}$ and $\left[D^{\prime}: E\right]=\left|h\left(\mathcal{E}^{\perp}\right)\right|$, 
$h\left(\mathcal{E}^{\perp}\right)$ is an armature of $D^{\prime}$ as an $E$-algebra. Furthermore, the image of $h(B)$ in $\Gamma_{D^{\prime}} / \Gamma_{E}$ is $\Gamma_{A} / \Gamma_{E}$. So, by the preceding paragraph $A \cong E[h(B)]=F[B]$, as desired.

Since $B=\bar{v}^{-1}\left(\Gamma_{A} / \Gamma_{F}\right)$, we have shown that $A$ is determined up to isomorphism by $\Gamma_{A} / \Gamma_{F}$. Furthermore, if $S$ is any subgroup fo $\Gamma_{D} / \Gamma_{F}, F\left[\bar{v}^{-1}(S)\right]$ is a subalgebra of $D$ and $\Gamma_{F\left[\bar{v}^{-1}(S)\right]} / \Gamma_{F}=S$. This gives the desired one-to-one correspondence. The rest of the theorem is immediate from (2.5)(i).

4. Division algebras over strictly Henselian fields. Let $(F, v)$ be a strictly Henselian valued field, i.e., suppose $v$ is a Henselian valuation on $F$ with $\bar{F}$ separably closed. If $D$ is a tame $F$-central division algebra, Draxl's analogue to Ostrowski's Theorem ((1.2) above) implies that $D$ is totally ramified over $F$ with respect to the unique extension of $v$ to $D$. We will show, in fact, that $D$ is determined up to isomorphism by $\Gamma_{D}$ and the canonical pairing $\Gamma_{D} / \Gamma_{F}$-see Proposition 4.2 below. The main result of this section is a method for determining the underlying division algebra of a tame central simple $F$-algebra.

Before considering division algebras over $F$, let us recall the classification of tame field extensions of $F$. As usual, $\Delta$ denotes the divisible hull of $\Gamma_{F}$.

Proposition 4.1. Suppose $(F, v)$ is a strictly Henselian valued field. For any tame field extension $L$ of $F, L$ is totally ramified and Galois over $F$ with $\mathcal{G}(L / F) \cong\left(\Gamma_{L} / \Gamma_{F}\right)$. The map $L \mapsto \Gamma_{L}$ gives a one-to-one inclusion-preserving correspondence between the tame field extensions of $F$ and all the subgroups $\Gamma$ of $\Delta$ with $\Gamma_{F} \subseteq \Gamma$ and $\operatorname{char}(\bar{F})+\left|\Gamma: \Gamma_{F}\right|<\infty$.

Proposition 4.1 follows easily from (1.4) above, in light of Ostrowski's theorem (the commutative version of (1.2) - see, e.g., [E, (20.21)]). The proposition says, in particular, that $L$ is determined up to isomorphism by $\Gamma_{L}$. For a tame $F$-central division algebra $D$ it is easy to see that $\Gamma_{D}$ does not completely determine $D$. We show that the further information about the noncommutative multiplication needed to specify $D$ completely is carried by the canonical pairing on $\Gamma_{D} / \Gamma_{F}$ defined in $\S 3$.

PROPOSITION 4.2. Let $(F, v)$ be a strictly Henselian valued field. Let $\Gamma$ be a subgroup of the divisible hull of $\Gamma_{F}$ with $\Gamma_{F} \subseteq \Gamma$ and $\operatorname{char}(\bar{F})+\left|\Gamma: \Gamma_{F}\right|<\infty$. Suppose $\Gamma / \Gamma_{F}$ admits a nondegenerate symplectic pairing $B$ into $\mu(\bar{F})$. Then there is a unique (up to isomorphism) division algebra $D$ tame over $F=Z(D)$ with $\Gamma_{D}=\Gamma$ and canonical pairing $C_{D}=B$. If $\left(\Gamma^{\prime}, B^{\prime}\right)$ is another such subgroup and nondegenerate symplectic pairing, then the corresponding $D^{\prime}$ is isomorphic to a subalgebra of $D$ if and only if $\Gamma^{\prime} \subseteq \Gamma$ and $B^{\prime}$ is the restriction of $B$ to $\Gamma^{\prime} / \Gamma_{F}$.

PARTIAL PROOF. For the moment we show how $D$ can be constructed from $\Gamma$ and $B$. Let $\left\{\bar{\alpha}_{1}, \bar{\beta}_{1}, \ldots, \bar{\alpha}_{k}, \bar{\beta}_{k}\right\}$ be a symplectic base of $\Gamma / \Gamma_{F}$ with respect to $B$. For $1 \leq i \leq k$, let $\bar{\omega}_{i}=B\left(\bar{\alpha}_{i}, \bar{\beta}_{i}\right) \in \mu(\bar{F})$, let $n_{i}=\operatorname{ord}\left(\bar{\alpha}_{i}\right)=\operatorname{ord}\left(\bar{\beta}_{i}\right)=\operatorname{ord}\left(\bar{\omega}_{i}\right)$, let $\omega_{i}$ be the unique inverse image of $\bar{\omega}_{i}$ in $\mu(F)$ with $\operatorname{ord}\left(\omega_{i}\right)=n_{i}$. Pick any inverse images $\alpha_{i}, \beta_{i} \in \Gamma$ of $\bar{\alpha}_{i}, \bar{\beta}_{i}$, and pick any $a_{i}, b_{i} \in F^{*}$ with $v\left(a_{i}\right)=n_{i} \alpha_{i}$ and $v\left(b_{i}\right)=n_{i} \beta_{i}$. Then set

$$
D=\bigotimes_{i=1}^{k} A_{\omega_{i}}\left(a_{i}, b_{i} ; F\right)
$$

Let $D$ be the obvious armature of $D$ (cf. 2.4(b) and (d)). We have $|D|=\left|\Gamma / \Gamma_{F}\right|$ and clearly $\Gamma / \Gamma_{F} \subseteq \bar{w}(D)$, where $\bar{w}$ is the map of Proposition 3.5. That proposition 
shows $D$ is a division algebra with $\Gamma_{D}=\Gamma$ and $C_{D}=B$, as desired. The remainder of the proof is deferred until after the proof of the next theorem.

Now, let $A$ be a tame central simple $F$-algebra. By Wedderburn's theorem $A$ is a matrix algebra, $A \cong M_{n}(D)$ for some division algebra $D$ with $Z(D)=F$ and $D$ tame over $F$. This $D$ is unique up to isomorphism; we call it the underlying division algebra of $A$. Over an arbitrary base field it seems to be a hopelessly difficult task to identify the underlying division algebra of a central simple $F$-algebra. Nonetheless, if $F$ is strictly Henselian and $A$ is tame we will now give a method for finding $D$, exploiting Draxl's decomposition Theorem 3.7 to see that $A$ has an armature.

THEOREM 4.3. Let $(F, v)$ be a strictly Henselian valued field, and let $A$ be any central simple $F$-algebra with $\operatorname{char}(\bar{F})+\operatorname{dim}_{F} A$. Let $A$ be any armature of $A$. Let $\mathcal{K} \subseteq A$ be the kernel of the homomorphism $\bar{w}: A \rightarrow \Delta / \Gamma_{F}$ of $(3.5)$, and $K^{\perp}$ the orthogonal dual of $K$ in $A$ with respect to the armature pairing $B_{A}$. If $D$ is the underlying division algebra of $A$, then $[D: F]=\left|K^{\perp}:\left(\mathcal{K} \cap \mathcal{K}^{\perp}\right)\right|, \Gamma_{D} / \Gamma_{F}=$ $\bar{w}\left(K^{\perp}\right) \subseteq \Delta / \Gamma_{F}$, and the canonical pairing on $\Gamma_{D} / \Gamma_{F}$ is isometric (via $\bar{w}$ ) to the pairing on $K^{\perp} /\left(K \cap K^{\perp}\right)$ induced by $B_{A}$. In particular,

(i) $A$ is a division algebra if and only if $\bar{w}$ is injective.

(ii) $A$ is split (i.e., $D=F$ ) if and only if $K \supseteq K^{\perp}$.

Proof. We have $A \cong M_{t}(D) \cong M_{t}(F) \otimes_{F} D$, for some $t$ with $\operatorname{char}(\bar{F}) \nmid t$. Since $\mu_{t} \subseteq F$ and Draxl's Theorem (3.7) applies to $D, A$ is a tensor product of symbol algebras; this assures the existence of an armature $A$ for $A$. Say $\operatorname{dim}_{F} A=n^{2}$.

Consider first the extreme case in which $K^{\perp} \subseteq K$. Then $K^{\perp}$ is totally isotropic, and there is a Lagrangian $\mathcal{L} \supseteq \mathcal{K}^{\perp}$. We have $|\mathcal{L}|=\sqrt{|\mathcal{A}|}=n$ and $\mathcal{K}^{\perp} \subseteq \mathcal{L}=$ $\mathcal{L}^{\perp} \subseteq K^{\perp \perp}=K$. Let $L=F[\mathcal{L}]$, which is commutative, as $\mathcal{L}$ is totally isotropic. Take any $\tilde{c}=c F^{*} \in \mathcal{L}$, and let $k=\operatorname{ord}(\tilde{c})$. Then, as $\mathcal{L} \subseteq \mathcal{K}, v\left(c^{k}\right) \in k \Gamma_{F}$; because $F$ is strictly Henselian and $\operatorname{char}(\bar{F}) \nmid k, c^{k} \in F^{* k}$. It follows by $(2.9(\mathrm{~b}))$ that $L \cong F \oplus \cdots \oplus F$ ( $n$ times). Since $A$ contains a family of $n$ orthogonal idempotents, by $\left[\mathbf{A}_{\mathbf{3}}\right.$, p. 50, Theorem 2] $A$ must be split, as desired.

Now consider the general case. Note that $B_{A}$ restricts to a symplectic pairing on $K^{\perp}$ whose radical is $K \cap K^{\perp}$. Hence $B_{A}$ induces a nondegenerate symplectic pairing on $K^{\perp} /\left(K \cap K^{\perp}\right)$. Let $\Gamma$ be the inverse image in $\Delta$ of $\bar{w}\left(K^{\perp}\right) \subseteq \Delta / \Gamma_{F}$, and let $\bar{w}^{\prime}: K^{\perp} /\left(\mathcal{K} \cap \mathcal{K}^{\perp}\right) \rightarrow \Gamma / \Gamma_{F}$ be the isomorphism induced by $\bar{w}$. Let $C$ be the nondegenerate symplectic pairing on $\Gamma / \Gamma_{F}$ with values in $\mu(\bar{F})$ induced via $\bar{w}^{\prime}$ by the pairing on $K^{\perp} /\left(K \cap K^{\perp}\right)$. From the part of Proposition 4.2 already proved there is a tame $F$-central division algebra $E$ with $\Gamma_{E}=\Gamma$ and $C$ for its canonical pairing. Let $\mathcal{E}$ be any armature of $E$, and $\bar{v}: \mathcal{E} \rightarrow \Gamma_{E} / \Gamma_{F}$ the isomorphism of (3.3). Let $g: K^{\perp} \rightarrow \mathcal{E}$ be the composition of surjective maps

$$
K^{\perp} \rightarrow K^{\perp} /\left(K \cap K^{\perp}\right) \stackrel{\bar{w}^{\prime}}{\longrightarrow} \Gamma_{E} / \Gamma_{F} \stackrel{\bar{v}^{-1}}{\longrightarrow} \mathcal{E} .
$$

Then, for any $\tilde{p}, \tilde{r} \in \mathcal{K}^{\perp}$,

$$
\bar{v}(g(\tilde{p}))=\bar{w}(\tilde{p}) \quad \text { and } \quad B_{\mathcal{E}}(g(\tilde{p}), g(\tilde{r}))=B_{A}(\tilde{p}, \tilde{r}) .
$$

We must show that $D \cong E$, where $D$ is the underlying division algebra of $A$. For this it suffices to verify that $A \otimes_{F} E^{\mathrm{op}}$ is split, where $E^{\mathrm{op}}$ is the opposite algebra of $E$. The elements of $\mathcal{E}$ form an armature of $E^{\mathrm{op}}$, which we denote $\mathcal{E}^{\prime}$ because $\mathcal{E}$ and $\mathcal{E}^{\prime}$ have different armature pairings: namely $B_{\mathcal{E}^{\prime}}(\tilde{e} . \tilde{f})=B_{\mathcal{E}}\left(\tilde{f}^{-1}, \tilde{e}^{-1}\right)=B_{\mathcal{E}}(\tilde{e}, \tilde{f})^{-1}$. 
Let $A \perp \mathcal{E}^{\prime}=\left\{\widetilde{a \otimes} e \mid \tilde{a} \in A\right.$ and $\left.\tilde{e} \in \mathcal{E}^{\prime}\right\}$, which is an armature for $A \otimes_{F} E^{\text {op }}$ (cf. $(2.4(\mathrm{~d}))$. For convenience, write $(\tilde{a}, \tilde{e})$ for the element $\widetilde{a \otimes e}$ of $A \perp \mathcal{E}^{\prime}$. Let $\bar{y}: A \perp \mathcal{E}^{\prime} \rightarrow \Delta / \Gamma_{F}$ be the map of (3.5), let $\mathcal{M}=\operatorname{ker}(\bar{y}) \subseteq A \perp \mathcal{E}^{\prime}$, and let $\mathcal{M}^{\perp}$ be the orthogonal dual of $\mathcal{M}$ with respect to $B_{A \perp \varepsilon^{\prime}}$. We will show that $\mathcal{M}^{\perp} \subseteq \mathcal{M}$; then the earlier case shows $A \otimes_{F} E^{\text {op }}$ is split.

Take any $(\tilde{a}, \tilde{e}) \in \mathcal{M}^{\perp}$. Since $g$ is surjective there is a $\tilde{p} \in \mathcal{K}^{\perp}$ with $g(\tilde{p})=\tilde{e}$. Now, take any $\tilde{r} \in \mathcal{K}^{\perp}$. Then $\bar{y}\left(\tilde{r}, g(\tilde{r})^{-1}\right)=\bar{w}(\tilde{r})+\bar{v}\left(g(\tilde{r})^{-1}\right)=0$ by $(*)$ above. So, $\left(\tilde{r}, g(\tilde{r})^{-1}\right) \in \mathcal{M}$, and we have, using $(*)$,

$$
\begin{aligned}
1 & =B_{A \perp \mathcal{E}^{\prime}}\left((\tilde{a}, \tilde{e}),\left(\tilde{r}, g(\tilde{r})^{-1}\right)\right)=B_{A}(\tilde{a}, \tilde{r}) \cdot B_{\mathcal{E}^{\prime}}\left(\tilde{e}, g(\tilde{r})^{-1}\right) \\
& =B_{A}(\tilde{a}, \tilde{r}) \cdot B_{\mathcal{E}}(g(\tilde{p}), g(\tilde{r}))=B_{A}(\tilde{a}, \tilde{r}) \cdot B_{A}(\tilde{p}, \tilde{r}) \\
& =B_{A}(\tilde{a} \tilde{p}, \tilde{r}) .
\end{aligned}
$$

Since this is true for all $\tilde{r} \in K^{\perp}$ we have $\tilde{a} \tilde{p} \in K^{\perp \perp}=K$. Thus,

$$
0=\bar{w}(\tilde{a} \tilde{p})=\bar{w}(\tilde{a})+\bar{w}(\tilde{p})=\bar{w}(\tilde{a})+\bar{v}(\tilde{e})=\bar{y}(\tilde{a}, \tilde{e}) .
$$

Hence $(\tilde{a}, \tilde{e}) \in \mathcal{M}$, which shows $\mathcal{M}^{\perp} \subseteq \mathcal{M}$. Therefore, $D \cong E$ as desired, and statements (i) and (ii) follow at once. (Of course, (i) was already known from (3.3) and (3.5).)

Proof of Proposition 4.2, COMPleted. Let $D_{1}$ and $D_{2}$ be two tame $F$ central division algebras with the same value group and the same canonical pairing. Then for any armature $D_{1}$ of $D_{1}$ we have by (3.3) and (3.4) that $\bar{v}: D_{1} \rightarrow \Gamma_{D_{1}} / \Gamma_{F}$ is an isomorphism and an isometry between $B_{D_{1}}$ and the canonical pairing of $D_{1}$. Then, the proof of Theorem 4.3 with $A=D_{1}$ and $E=D_{2}$ shows that $D_{2}$ is the underlying division algebra of $D_{1}$, i.e., $D_{2} \cong D_{1}$. This gives the uniqueness part of (4.2).

Now take any two tame $F$-central division algebras $D$ and $D^{\prime} ;$ suppose $\Gamma_{D^{\prime}} \subseteq \Gamma_{D}$ and the canonical pairing of $D$ restricts to the canonical pairing of $D^{\prime}$. Let $D$ be any armature of $D$, and $\bar{v}: D \rightarrow \Gamma_{D} / \Gamma_{F}$ the isomorphism of (3.3). Let $D^{\prime}=$ $\bar{v}^{-1}\left(\Gamma_{D^{\prime}} / \Gamma_{F}\right) \subseteq D$. Then $F\left[D^{\prime}\right]$ and $D^{\prime}$ have the same value group and canonical pairing. By what we just proved, $D^{\prime} \cong F\left[D^{\prime}\right] \subseteq D$, as desired. Since the converse part of the last statement of (4.2) is trivial, (4.2) is now fully proved.

Here are some further applications of the theorem. We continue to assume that $(F, v)$ is strictly Henselian.

EXAMPLES 4.4. (i) Let $D_{1}$ and $D_{2}$ be two $F$-central division algebras, with value groups $\Gamma_{1}$ and $\Gamma_{2}$ and canonical pairings $C_{1}$ and $C_{2}$. We determine the underlying division algebra $D$ of $D_{1} \otimes_{F} D_{2}$. Let $\Phi=\left(\Gamma_{1} / \Gamma_{F}\right) \times\left(\Gamma_{2} / \Gamma_{F}\right)$. Then $C_{1} \perp C_{2}$ is a nondegenerate symplectic pairing on $\Phi$, and there is a homomorphism $f: \Phi \rightarrow \Delta / \Gamma_{F}$ given by $(\bar{\alpha}, \bar{\beta}) \mapsto \bar{\alpha}+\bar{\beta}$. Let $K=\operatorname{ker}(f) \subseteq \Phi$ and $K^{\perp}$ the orthogonal dual of $K$ in $\Phi$ with respect to $C_{1} \perp C_{2}$. Then $[D: F]=\left|K^{\perp}:\left(K \cap K^{\perp}\right)\right|$, $\Gamma_{D} / \Gamma_{F}=f\left(K^{\perp}\right)$, and the canonical pairing on $\Gamma_{D} / \Gamma_{F}$ is isometric (via $f$ ) to the pairing on $K^{\perp} /\left(K \cap K^{\perp}\right)$ induced by $C_{1} \perp C_{2}$ on $\Phi$. This is easily verified from the theorem using the isometry between the canonical pairings $C_{i}$ and the armature pairings $B_{D_{i}}$ for any armature $D_{i}$ of $D_{i}, i=1,2$, together with Example 2.4(d) above.

(ii) Consider now a tame $F$-central division algebra $D$ with canonical pairing $C_{D}$, and let $D^{\prime}$ be the underlying division algebra of $D \otimes_{F} D \otimes_{F} \cdots \otimes_{F} D$, the $m$ th tensor power of $D$, for any natural number $m$. By the same approach as for 
(i) (or by repeated applications of (i)), one can see that $D^{\prime}$ is the tame $F$-division algebra with $\left[D^{\prime}: F\right]=\left|\left(m \Gamma_{D}+\Gamma_{F}\right): \Gamma_{F}\right|, \Gamma_{D^{\prime}}=m \Gamma_{D}+\Gamma_{F}$, and the canonical pairing on $\Gamma_{D^{\prime}}$ is given by $C_{D^{\prime}}(m \bar{\alpha}, m \bar{\beta})=C_{D}(\bar{\alpha}, \bar{\beta})^{m}$ for all $\bar{\alpha}, \bar{\beta} \in \Gamma_{D} / \Gamma_{F}$. Thus, the order of $[D]$ in the Brauer group $\operatorname{Br}(F)$ is clearly $\exp \left(\Gamma_{D} / \Gamma_{F}\right)$. (This formula for the order of $[D]$ can also be verified easily using the decomposition of $D$ into symbol algebras.)

The theorem also allows us to determine what happens to $D$ upon extension of scalars:

Proposition 4.5. Let $(F, v)$ be a strictly Henselian valued field, and $D$ a tame $F$-central division algebra with canonical pairing $C_{D}$. Let $\Delta$ be the divisible hull of $\Gamma_{F}$. Let $E$ be any finite degree field extension of $F$, and let $D^{\prime}$ be the underlying division algebra of $D \otimes_{F} E$. Let $\Psi=\left(\Gamma_{D} \cap \Gamma_{E}\right) / \Gamma_{F} \subseteq \Delta / \Gamma_{F}$, and $\Psi^{\perp}$ the orthogonal dual of $\Psi$ in $\Gamma_{D} / \Gamma_{F}$ with respect to $C_{D}$. Then $\left[D^{\prime}: E\right]=$ $\left|\Psi^{\perp}:\left(\Psi \cap \Psi^{\perp}\right)\right|, \Gamma_{D^{\prime}} / \Gamma_{E}$ is the image of $\Psi^{\perp}$ in $\left(\Gamma_{D}+\Gamma_{E}\right) / \Gamma_{E}$, and the canonical pairing on $\Gamma_{D^{\prime}} / \Gamma_{E}$ is isometric to the pairing on $\Psi^{\perp} /\left(\Psi \cap \Psi^{\perp}\right)$ induced by $C_{D}$. In particular,

(i) $E$ splits $D$ if and only if $\Psi \supseteq \Psi^{\perp}$.

(ii) $D \otimes_{F} E$ is a division algebra if and only if $\Gamma_{E} \cap \Gamma_{D}=\Gamma_{F}$.

ProOF. Note that $E$ is strictly Henselian with respect to the unique extension of $v$ to $E$, and $D \otimes_{F} E$ is a tame $E$-algebra. Let $D$ be any armature of $D$, and $\bar{v}: D \rightarrow \Gamma_{D} / \Gamma_{F}$ the isomorphism induced by $v$. Now, $D_{E}:=\{\widetilde{d \otimes} 1 \mid \tilde{d} \in D\} \subseteq$ $\left(D \otimes_{F} E\right)^{*} / E^{*}$ is an armature of $D \otimes_{F} E$ as an $E$-algebra (cf. (2.4)(e)), and the natural map $f: D \rightarrow D_{E}$ is an isomorphism which is also an isometry between the armature pairings $B_{D}$ and $B_{D_{E}}$. Let $g: \Delta / \Gamma_{F} \rightarrow \Delta / \Gamma_{E}$ be the natural projection, let $\bar{w}: D_{E} \rightarrow \Delta / \Gamma_{E}$ be the map of (3.5), and let $K=\operatorname{ker}(\bar{w})$. Since $\bar{w} \circ f=g \circ \bar{v}$, we have $\bar{v} \circ f^{-1}(\mathcal{K})=\operatorname{ker}(g) \cap\left(\Gamma_{D} / \Gamma_{F}\right)=\Psi$. Note that $\bar{v} \circ f^{-1}$ is an isometry between $B_{D_{E}}$ on $D_{E}$ and $C_{D}$ on $\Gamma_{D} / \Gamma_{F}$. Hence, $\bar{v} \circ f^{-1}\left(K^{\perp}\right)=\Psi^{\perp}$, and the proposition follows immediately from Theorem 4.3.

This proposition yields an easy nonhomological proof of the main result of [ $\left.\mathbf{T A}_{\mathbf{1}}\right]$ :

Corollary 4.6 (Tignol, Amitsur). Let $F, v, D$, and $E$ be as in Proposition 4.5. Then $E$ splits $D$ if and only if $E$ contains an isomorphic copy of $a$ maximal subfield of $D$.

Proof. Suppose $E$ splits $D$. Let $E_{0}$ be the maximal tame extension of $F$ in $E$. Then $E_{0}$ splits $D$ since $\left[E: E_{0}\right]$ is a power of $\operatorname{char}(\bar{F})$ which is prime to $[D: F]$. Let $\Psi=\left(\Gamma_{E_{0}} \cap \Gamma_{D}\right) / \Gamma_{F}$. By Proposition $4.5, \Psi \supseteq \Psi^{\perp}$. Hence, $\Psi^{\perp}$ is totally isotropic with respect to the pairing $C_{D}$ on $\Gamma_{D} / \Gamma_{F}$, and we can find a Lagrangian $\Lambda$ of $\Gamma_{D} / \Gamma_{F}$ with $\Psi^{\perp} \subseteq \Lambda$. Then $\Psi^{\perp} \subseteq \Lambda=\Lambda^{\perp} \subseteq \Psi$. Let $L$ be the subalgebra of $D$ associated to $\Lambda$ in the correspondence of (3.8). Then $\Gamma_{L} / \Gamma_{F}=\Lambda$ and, since $\Lambda$ is a Lagrangian of $\Gamma_{D} / \Gamma_{F}, L$ is a maximal subfield of $D$. Since $\Gamma_{L} / \Gamma_{F} \subseteq \Psi \subseteq \Gamma_{E_{0}} / \Gamma_{F}$, Proposition 4.1 shows that $L$ is isomorphic to a subfield of $E_{0}$, hence of $E$, as desired. The converse is clear.

Using the extension Lemma 1.6 we can carry over some of the information obtained in the strictly Henselian case to totally ramified division algebras over an arbitrary field. 
THEOREM 4.7. Let $(D, v)$ be a valued division algebra which is tame and totally ramified over its center $F$, which can be any valued field. Let $C_{D}$ be the canonical pairing on $\Gamma_{D} / \Gamma_{F}$. Then,

(i) $\exp \left(\Gamma_{D} / \Gamma_{F}\right)$ divides the order of $[D]$ in $\operatorname{Br}(F)$.

(ii) Let $K \supseteq F$ be any field, and let $D^{\prime}$ be the underlying division algebra of $D \otimes_{F} K$. If $w$ is any extension of $v$ to $K$, then $\left[D^{\prime}: K\right]$ is a multiple of $\mid \Psi^{\perp}$ : $\left(\Psi \cap \Psi^{\perp}\right) \mid$, where $\Psi=\left(\Gamma_{K} \cap \Gamma_{D}\right) / \Gamma_{F}$ and $\Psi^{\perp} \subseteq \Gamma_{D} / \Gamma_{F}$ is taken relative to $C_{D}$. Equality holds if $w$ extends to a valuation on $D^{\prime}$ totally ramified over $K$.

(iii) For $(K, w)$ as in (ii) with $[K: F]<\infty$ suppose $K$ splits $D$. Then

$$
\operatorname{rank}\left(\Gamma_{K} / \Gamma_{F}\right) \geq \frac{1}{2} \operatorname{rank}\left(\Gamma_{D} / \Gamma_{F}\right) \text {. }
$$

If $K$ is Galois over $F$, then $\mathcal{G}(K / F)$ has a subgroup isomorphic to a Lagrangian of $\Gamma_{D} / \Gamma_{F}$.

(iv) If

$$
[D]=\left[\bigotimes_{i=1}^{k} A_{\omega_{i}}\left(b_{i}, c_{i} ; F\right)\right]
$$

in the Brauer group $\operatorname{Br}(F)$, then $k \geq \frac{1}{2} \operatorname{rank}\left(\Gamma_{D} / \Gamma_{F}\right)$.

(v) In particular, if $\operatorname{rank}\left(\Gamma_{D} / \Gamma_{F}\right)>2$, then $D$ is not split by any cyclic Galois extension of $F$.

ProOF. Let $F_{s}$ be a separable closure of $F$, and $v_{s}$ any extension of $v$ to $F_{s}$. Let $F^{\prime}$ be the inertia field of $v_{s}$ over $v$ (cf. [E, §19]), and $v^{\prime}=v_{s} \mid F^{\prime}$. Then $\left(F^{\prime}, v^{\prime}\right)$ is strictly Henselian and $\Gamma_{F^{\prime}}=\Gamma_{F}$. By the extension lemma 1.6, $D_{1}:=D \otimes_{F} F^{\prime}$ is a division ring with $\Gamma_{D_{1}}=\Gamma_{D}$. Thus, using 4.4(ii), we have $\exp \left(\Gamma_{D} / \Gamma_{F}\right)=$ $\exp \left(\Gamma_{D_{1}} / \Gamma_{F^{\prime}}\right)=\operatorname{ord}\left[D_{1}\right]$. Since ord $\left[D_{1}\right] \mid \operatorname{ord}[D]$, this yields (i).

Now, given $(K, w)$ as in (ii), construct $\left(K_{s}, w_{s}\right)$ and $\left(K^{\prime}, w^{\prime}\right)$ in the same manner as we just constructed $\left(F_{s}, v_{s}\right)$ and $\left(F^{\prime}, v^{\prime}\right)$ from $(F, v)$. We may assume that $F_{s} \subseteq$ $K_{s}$ and $v_{s}=\left.w_{s}\right|_{F_{s}}$; so $F^{\prime} \subseteq K^{\prime}$. Let $D_{2}$ be the underlying division algebra of $D^{\prime} \otimes_{K} K^{\prime}$ (which is also the underlying division algebra of $D_{1} \otimes_{F^{\prime}} K^{\prime}$ ). Since $\Psi=\left(\Gamma_{K} \cap \Gamma_{D}\right) / \Gamma_{F}=\left(\Gamma_{K^{\prime}} \cap \Gamma_{D_{1}}\right) / \Gamma_{F^{\prime}}$, we have

$$
\left|\Psi^{\perp}:\left(\Psi \cap \Psi^{\perp}\right)\right|=\left[D_{2}: K^{\prime}\right]
$$

(which divides $\left[D: K^{\prime}\right]$ ) by the argument for Proposition 4.5 applied to $K^{\prime}$ over $F^{\prime}$. (The proof of (4.5) uses $E$ and $F$ strictly Henselian, but does not require $[E: F]<\infty$.) If $w$ extends to a valuation on $D^{\prime}$ totally ramified over $K$, then the extension lemma shows $D_{2}=D^{\prime} \otimes_{K} K^{\prime}$, hence $\left[D^{\prime}: K\right]=\left[D_{2}: K^{\prime}\right]$, completing the proof of (ii).

For (iii), suppose $K$ splits $D$. Then (ii) shows $\Psi^{\perp} \subseteq \Psi$. Hence there is a Lagrangian $\Lambda$ of $\Gamma_{D} / \Gamma_{F}$ with $\Lambda \subseteq \Psi \subseteq \Gamma_{K} / \Gamma_{F}$, which yields $\operatorname{rank}\left(\Gamma_{K} / \Gamma_{F}\right) \geq$ $\operatorname{rank}(\Lambda) \geq \frac{1}{2} \operatorname{rank}\left(\Gamma_{D} / \Gamma_{F}\right)\left(\operatorname{cf}\right.$. (2.3)). If $K$ is Galois over $F$, then $K_{s}=F_{s}$ and $K^{\prime}=F^{\prime} \cdot K$. Hence $K^{\prime}$ is Galois over $F^{\prime}$ and $\mathcal{G}\left(K^{\prime} / F^{\prime}\right)$ injects into $\mathcal{G}(K / F)$. Let $M$ be the ramification field of $w^{\prime}$ over $v^{\prime}$. Then $F^{\prime} \subseteq M \subseteq K^{\prime}, M$ is Galois over $F^{\prime}$, and $\mathcal{G}\left(K^{\prime} / M\right)$ is the unique $p$-Sylow subgroup of $\mathcal{G}\left(K^{\prime} / F^{\prime}\right)$ where $p=\operatorname{char}(\bar{F})$ (cf. $[\mathbf{E},(20.11)])$. Because $K^{\prime}$ splits $D$ and $\left[K^{\prime}: M\right]$ is prime to $[D: F], D$ must already be split by $M$. The argument just given shows $\Gamma_{M} / \Gamma_{F}$ contains a Lagrangian $\Lambda$ of $\Gamma_{D} / \Gamma_{F}$. But $\mathcal{G}\left(M / F^{\prime}\right) \cong \Gamma_{M} / \Gamma_{F^{\prime}}$ as $M$ is tame over $F^{\prime}$ which is strictly Henselian. Furthermore, by the Schur-Zassenhaus lemma [Ro, p. 151] since 
$\mathcal{G}\left(K^{\prime} / M\right)$ is normal in $\mathcal{G}\left(K^{\prime} / F^{\prime}\right)$ and since $\mathcal{G}\left(K^{\prime} / M\right)$ and $\mathcal{G}\left(M / F^{\prime}\right)$ have relatively prime orders, $\mathcal{G}\left(M / F^{\prime}\right)$ is isomorphic to a subgroup of $\mathcal{G}\left(K^{\prime} / F^{\prime}\right)$. Thus we have a succession of injective maps $\Lambda \hookrightarrow \Gamma_{M} / \Gamma_{F^{\prime}} \hookrightarrow \mathcal{G}\left(M / F^{\prime}\right) \hookrightarrow \mathcal{G}\left(K^{\prime} / F^{\prime}\right) \hookrightarrow \mathcal{G}(K / F)$, proving (iii).

Now suppose $[D]=\left[\bigotimes_{i=1}^{k} A_{\omega_{i}}\left(b_{i}, c_{i} ; F\right)\right]$ in $\operatorname{Br}(F)$. Let $n_{i}=\operatorname{ord}\left(\omega_{i}\right)$ in $F^{*}$, and let $K$ be the field $F\left(b_{1}^{1 / n_{1}}, \ldots, b_{k}^{1 / n_{k}}\right)$. Then $K$ is a Kummer extension of $F$, and $K$ splits $D$ since it splits each of the symbol algebras. Because $\mathcal{G}(K / F)$ contains a Lagrangian $\Lambda$ of $\Gamma_{D} / \Gamma_{F}$ by (iii), we have $k \geq \operatorname{rank}(\mathcal{G}(K / F)) \geq \operatorname{rank}(\Lambda) \geq$ $\frac{1}{2} \operatorname{rank}\left(\Gamma_{D} / \Gamma_{F}\right)$, proving (iv).

Part (v) is immediate from (iii) since if $\operatorname{rank}\left(\Gamma_{D} / \Gamma_{F}\right)>2, \Gamma_{D} / \Gamma_{F}$ has no cyclic Lagrangians.

Parts (iii) and (iv) of Theorem 4.7 are generalizations of [ $\mathbf{T A}_{\mathbf{2}}$, Theorem 4.4] and the proof of [ $\mathbf{T}_{\mathbf{2}}$, Lemma 4.1].

REMARK 4.11. The Brauer group $\operatorname{Br}(F)$ of a strictly Henselian field $F$ is known (except for the char $(\bar{F})$-primary component), and has a nice homological description (cf. [Sch, or $\left.\mathbf{W}_{\mathbf{1}}\right]$ ). Bill Jacob suggested to us that there ought also to be a homological interpretation of the process for finding the underlying division algebra in Theorem 4.3. From this perspective the theorem turns out to be equivalent to a result on exterior products of $\mathbf{Z}$-modules. We now sketch this approach.

Let $(F, v)$ be a strictly Henselian valued field, and let $p$ be a prime number, $p \neq \operatorname{char}(\bar{F})$. So, $\mu_{p^{n}} \subseteq F$ for all $n$. Let $\tilde{F}^{p}$ be the $p$-closure of $F$, which is the compositum of all finite-degree Galois extensions $K$ of $F$ with $[K: F]$ a power of $p$. Then $\tilde{F}^{p}$ is a Galois extension of $F$ with pro- $p$ Galois group $G:=\mathcal{G}\left(\tilde{F}_{p} / F\right)$. Because $F$ is strictly Henselian $G$ is a free abelian pro- $p$ group. Also, since $\tilde{F}_{p}$ has no field extension of $p$-power degree the relative Brauer group $\operatorname{Br}\left(\tilde{F}_{p} / F\right)\left(\cong H^{2}\left(G, \tilde{F}_{p}^{*}\right)\right)$ is the full $p$-primary component of $\operatorname{Br}(F)$. Hence, for each $n$ the continuous cohomology group $H^{2}\left(G, \mu_{p^{n}}\right)$ is the full $p^{n}$-torsion subgroup of $\operatorname{Br}(F)$.

Because $G$ is free abelian, the cup product pairing

$$
\cup: H^{1}\left(G, \mu_{p^{n}}\right) \times H^{1}\left(G, \mu_{p^{n}}\right) \rightarrow H^{2}\left(G, \mu_{p^{n}}\right)
$$

induces an isomorphism

$$
H^{1}\left(G, \mu_{p^{n}}\right) \wedge H^{1}\left(G, \mu_{p^{n}}\right) \cong H^{2}\left(G, \mu_{p^{n}}\right),
$$

where $\wedge$ denotes the exterior product (as $\mathbf{Z}$-modules). Of course, as $\mu_{p^{n}} \subseteq F$,

$$
H^{1}\left(G, \mu_{p^{n}}\right) \cong F^{*} / F^{* p^{n}} \cong \Gamma_{F} / p^{n} \Gamma_{F}
$$

Finding the underlying division algebra of a $\gamma \in H^{2}\left(G, \mu_{p^{n}}\right)$ corresponds to finding a minimal representation of $\gamma$ in the form $\gamma=\sum_{i=1}^{k} \alpha_{i} \wedge \beta_{i}$ with $\alpha_{i}, \beta_{i} \in H^{1}\left(G, \mu_{p^{n}}\right)$ (minimal in the sense that $\sum_{i=1}^{k} \operatorname{ord}\left(\alpha_{i} \wedge \beta_{i}\right)$ is minimal). The method for finding $D$ in Theorem 4.3 generalizes to give an algorithm for finding a minimal representation of any element of $M \wedge M$ (exterior product as $R / I$-modules) where $M$ is any $R / I$ module, for $R$ a principal ideal domain (or Dedekind domain) and $I$ any nonzero ideal of $R$. As we describe this more explicitly, we restrict for simplicity to the case where $R / I$ is a field.

Suppose, then, that $M$ is a vector space of any dimension over any field $F$. Take any $\gamma \in M \wedge M$. A minimal representation of $\gamma$ is an expression $\gamma=\sum_{i=1}^{k} \alpha_{i} \wedge \beta_{i}$ 
with $k$ minimal. The representation is easily seen to be minimal if and only if $B:=$ $\left\{\alpha_{1}, \beta_{1}, \ldots, \alpha_{k}, \beta_{k}\right\}$ is linearly independent over $F$. When this linear independence holds, let $V_{B}=\operatorname{span} B$, and let $B_{B}: V \times V \rightarrow F$ be the nondegenerate symplectic $F$-bilinear pairing on $V$ with symplectic base $B$. It is presumably well-known that $\sum_{i=1}^{k} \alpha_{i}^{\prime} \wedge \beta_{i}^{\prime}$ is another minimal representation of $\gamma$ if and only if $\left\{\alpha_{i}^{\prime}, \beta_{i}^{\prime}, \ldots, \alpha_{k}^{\prime}, \beta_{k}^{\prime}\right\}$ is another symplectic base of $B_{B}$ on $V_{B}$. Thus, the elements of $M \wedge M$ are in 1-1 correspondence with pairs $(V, B)$, where $V$ is an even (finite) dimensional subspace of $M$ and $B$ is a nondegenerate symplectic pairing on $V$.

Now, suppose we have a (possibly nonminimal) representation $\gamma=\sum_{i=1}^{l} \delta_{i} \wedge \varepsilon_{i}$ of $\gamma \in M \wedge M$. The method for finding $(V, B)$ corresponding to $\gamma$ analogous to Theorem 4.3 runs as follows: Let $C=\left\{x_{1}, y_{1}, \ldots, x_{l}, y_{l}\right\}$ be a base of a vector space $W$, let $B_{W}$ be the symplectic pairing on $W$ with $C$ as a symplectic base, and let $f: W \rightarrow M$ be the $F$-linear transformation given by $x_{i} \mapsto \delta_{i}$ and $y_{i} \mapsto \varepsilon_{i}, 1 \leq i \leq l$. Let $K=\operatorname{ker}(f)$ and $K^{\perp}$ be the orthogonal dual of $K$ with respect to $B_{W}$. Then $V=f\left(K^{\perp}\right)$ and $B$ is the symplectic pairing on $V$ isometric (via $f$ ) to the one induced on $K^{\perp} /\left(K \cap K^{\perp}\right)$ by $B_{W}$. The proof is omitted.

5. Examples. As applications of the theory developed in the preceding sections, we now construct two examples.

The first example is a pair of division algebras $D_{1}, D_{2}$ central simple over a field $F$ such that $D_{1} \otimes_{F} D_{2}$ is not a division algebra but $D_{1}$ and $D_{2}$ have no common subfield properly containing $F$. This answers an old question dating back to Albert's work in $\left[\mathbf{A}_{1}\right]$. Albert showed that if $Q_{1}$ and $Q_{2}$ are quaternion algebras over $F$ and $Q_{1} \otimes Q_{2}$ is not a division algebra, then $Q_{1}$ and $Q_{2}$ have a common maximal subfield. Of course, it was not really expected that Albert's result would extend to arbitrary finite-dimensional division algebras. The main obstacle in producing a counterexample has been the difficulty in determining all the subfields of a given division algebra.

For this example, let $n$ be any odd integer, $n>1$, and let $k$ be any field containing a primitive $n$th root of unity $\omega$. Let $F=k(t, x, y)$, where $t, x, y$ are independent indeterminates over $k$. Let

$$
D_{1}:=A_{\omega}(x, y ; F) \quad \text { and } \quad D_{2}:=A_{\omega}(x(t-1) / y, x t ; F) .
$$

Proposition 5.1. $\quad D_{1}$ and $D_{2}$ are division algebras, $D_{1} \otimes_{F} D_{2}$ is not a division algebra, but there is no field $K \supsetneqq F$ isomorphic to a subfield of $D_{1}$ and also to a subfield of $D_{2}$.

PROOF. Let $z=x(t-1) / y$ and $\pi=x t=x+y z$. In $D_{1} \otimes_{F} D_{2}$ we have elements $i_{1}, j_{1}, i_{2}, j_{2}$ which commute pairwise except that $i_{1} j_{1}=\omega j_{1} i_{1}$ and $i_{2} j_{2}=\omega j_{2} i_{2}$; further $i_{1}^{n}=x, j_{1}^{n}=y, i_{2}^{n}=z, j_{2}^{n}=\pi$. Let $\alpha=j_{1} i_{1}^{-1} i_{2}, \gamma=i_{1} j_{2}^{-1}$, and $\beta=(1+\alpha) \gamma=\left(i_{1}+j_{1} i_{2}\right) j_{2}^{-1}$. Since $\gamma \alpha \gamma^{-1}=\omega^{2} \alpha$, we have

$$
\begin{aligned}
\beta^{n} & =(1+\alpha) \gamma(1+\alpha) \gamma^{-1} \gamma^{2}(1+\alpha) \gamma^{-2} \cdots \gamma^{n-1}(1+\alpha) \gamma^{-(n-1)} \gamma^{n} \\
& =(1+\alpha)\left(1+\omega^{2} \alpha\right) \cdots\left(1+\omega^{2(n-1)} \alpha\right) \gamma^{n} .
\end{aligned}
$$

Recall the cyclotomic identity $X^{n}-1=\prod_{i=0}^{n-1}\left(X-\rho^{i}\right)$, for any primitive $n$th root of unity $\rho$. Since $n$ is odd, this yields $X^{n}+1=-\left[(-X)^{n}-1\right]=\prod_{i=0}^{n-1}\left(X+\rho^{i}\right)$. 
Since $\rho=\omega^{-2}$ is a primitive $n$th root of unity, we have

$$
\prod_{i=0}^{n-1}\left(1+\omega^{2 i} \alpha\right)=\left(\omega^{2}\right)^{n(n-1) / 2} \prod_{i=0}^{n-1}\left(\omega^{-2 i}+\alpha\right)=1 \cdot\left(1+\alpha^{n}\right) .
$$

Therefore,

$$
\beta^{n}=\left(1+\alpha^{n}\right)\left(i_{1} j_{2}^{-1}\right)^{n}=\left(1+y x^{-1} z\right)\left(x \pi^{-1}\right)=(x+y z) \pi^{-1}=1 .
$$

Since $\beta$ is not central in $D_{1} \otimes_{F} D_{2}, \beta \notin F$. Thus, $F(\beta)$ cannot be a field, since it contains $n+1$ different $n$th roots of unity. Therefore, $D_{1} \otimes_{F} D_{2}$ is not a division ring.

(Alternatively, one can check, using the identities for symbol algebras, that in the Brauer group $\operatorname{Br}(F)$

$$
\begin{aligned}
{\left[D_{1} \otimes_{F} D_{2}\right] } & =\left[A_{\omega}(x, y ; F)\right]+\left[A_{\omega}(x(t-1) / y, x t ; F)\right] \\
& =\left[A_{\omega}\left(x, t y^{2} /(t-1) ; F\right)\right]+\left[A_{\omega}(t, y ; F)\right]=\left[A_{\omega}\left(x t^{s}, t y^{2} /(t-1) ; F\right)\right],
\end{aligned}
$$

where $s=(n+1) / 2$, an integer.)

It remains to see that $D_{1}$ and $D_{2}$ are division algebras with "no" common subfields. For this, let $F^{\prime}=k(t)((x))((y))$, the twice iterated Laurent power series field over $k(t)$, and let $D_{i}^{\prime}=D_{i} \otimes_{F} F^{\prime}$. It suffices to see that $D_{1}^{\prime}$ and $D_{2}^{\prime}$ are division algebras with "no" common subfields. For, if $K \supsetneqq F$ is a field embedding in $D_{1}$ and $D_{2}$ then $K \otimes_{F} F^{\prime}$ embeds in $D_{1}^{\prime}$ and $D_{2}^{\prime}$. The embedding in the $D_{i}^{\prime}$ assures that $K \otimes_{F} F^{\prime}$ is a field.

Let $v$ be the standard Henselian valuation on $F^{\prime}$, as described in (3.6) above. So, $\overline{F^{\prime}}=k(t), \Gamma_{F^{\prime}}=\mathbf{Z} \times \mathbf{Z}$, and $v(x)=(1,0), v(y)=(0,1)$. Because $v(x), v(y)$ map onto a base of $\Gamma_{F} / n \Gamma_{F},(3.5)$ (or [ $\mathbf{J W}$, Corollary 2.6]) shows that $D_{1}^{\prime}=A_{\omega}\left(x, y ; F^{\prime}\right)$ is a division algebra and the extension of $v$ to $D_{1}^{\prime}$ is totally ramified over $F^{\prime}$, with $\Gamma_{D_{1}^{\prime}}=(1 / n) \mathbf{Z} \times(1 / n) \mathbf{Z}$. By Theorem 3.8 the subfields $L$ of $D_{1}^{\prime}, L \supsetneqq F^{\prime}$ are classified by the nonidentity totally isotropic subgroups of $\Gamma_{D_{1}^{\prime}} / \Gamma_{F^{\prime}}$. The minimal proper field extensions of $F^{\prime}$ in $D_{1}^{\prime}$ correspond to minimal nonidentity subgroups of $\Gamma_{D_{1}^{\prime}} / \Gamma_{F^{\prime}}$. Every such minimal subgroup is generated by $(i / p, j / p)(\bmod \mathbf{Z} \times \mathbf{Z})$ for some prime $p$ dividing $n$ and integers $i, j$, not both mutiples of $p$; the corresponding field must be $F\left(\sqrt[p]{x^{i} y^{j}}\right)$ since this is a subfield of $D_{1}^{\prime}$ with the right value group.

Now, $D_{2}^{\prime}=A_{\omega}\left(z, \pi ; F^{\prime}\right)$ where $z=x(t-1) / y$ and $\pi=x t$. Since $v(z)=(1,-1)$ and $v(\pi)=(1,0)$, the same analysis for $D_{1}^{\prime}$ shows $D_{2}^{\prime}$ is a division algebra, the extension of $v$ from $F^{\prime}$ to $D_{2}^{\prime}$ is totally ramified with $\Gamma_{D_{2}^{\prime}}=(1 / n) \mathbf{Z} \times(1 / n) \mathbf{Z}$, and every subfield $L$ of $D_{2}^{\prime}, L \supsetneqq F^{\prime}$ contains a subfield of the form $F^{\prime}\left(\sqrt[p]{z^{l} \pi^{m}}\right)$ with $p \mid n$ prime and $l, m \in \mathbf{Z}$, not both multiples of $p$. Thus, if $D_{1}^{\prime}$ and $D_{2}^{\prime}$ have a common subfield we must have $F^{\prime}\left(\sqrt[p]{x^{i} y^{j}}\right)=F^{\prime}\left(\sqrt[p]{z^{l} \pi^{m}}\right) \supsetneqq F^{\prime}$ for suitable $p, i, j, l, m$. By Kummer theory $z^{l} \pi^{m} \equiv\left(x^{i} y^{j}\right)^{q}\left(\bmod F^{* p}\right)$, for some integer $q$. By comparing the values of these expressions we find $(i q, j q) \equiv(l+m,-l)(\bmod p \mathbf{Z} \times p \mathbf{Z})$, i.e., $i q \equiv(l+m)(\bmod p)$ and $j q \equiv-l(\bmod p)$. Hence $F^{* * p}$ contains $z^{l} \pi^{m} x^{-(l+m)} y^{l}=$ $(t-1)^{l} t^{m}$. This unit must then map to a $p$ th power in the residue field $\overline{F^{\prime}}=k(t)$. Hence, $p \mid l$ and $p \mid m$, so that $F^{\prime}\left(\sqrt[p]{z^{l} \pi^{m}}\right)=F^{\prime}$, a contradiction.

REMARK. David Saltman has recently communicated the following observation about this example: Although $D_{1} \otimes_{F} D_{2}$ is not a division algebra, $D_{1}^{\mathrm{op}} \otimes_{F} D_{2}$ is a division algebra. (For, by the identities for symbol algebras, $D_{1}^{\mathrm{op}} \otimes_{F} D_{2} \cong$ 
$A_{\omega}((t-1) / t, x ; F) \otimes_{F} A_{\omega}(t, y ; F)$. By $[\mathbf{J W}$, Corollary 2.9$]$ the latter tensor product is a division algebra since it has a valuation (whose restriction to $F$ coincides with the valuation $F$ inherits from $F^{\prime}$ ).) As $D_{1}$ and $D_{1}^{\text {op }}$ have the same subfields, there can be "no" common subfield to $D_{1}$ and $D_{2}$. Saltman raises the following question: If $D_{1}$ and $D_{2}$ are any $F$-central division algebras of prime index $p$ and if $D_{1} \otimes_{F} D_{2}^{(i)}$ is not a division algebra for $1 \leq i \leq p-1$ (where $D_{2}^{(i)}$ is the underlying division algebra of the $i$ th tensor power of $D_{2}$ ), must $D_{1}$ and $D_{2}$ have a common subfield? Albert's theorem says yes if $p=2$. But for $p \geq 3$ the question is open.

Our second example is a division algebra $D$ which is not expressible as a tensor product of symbol algebras. The first such algebra was constructed by Albert in $\left[\mathbf{A}_{2}\right]$. Also, it is known that the generic division algebra $U D(F, n)$ is not a tensor product of symbol algebras if $p^{2} \mid n$ for some prime $p$. $(U D(F, n)$ is the quotient division algebra of the $F$-algebra generated by at least two generic $n \times n$ matrices over $F$.) What is new here is the method of construction: We will arrange to have different valuations on $D$, so that no possible tensor decomposition of $D$ is compatible with all the relative value groups. The approach of playing off one valuation against another seems very useful for building counterexamples. This method was employed in the construction of noncrossed products in [JW].

Let $r$ and $s$ be any integers, $r>1, s>1$. We begin with fields $F \subseteq K$, with $\mu_{r s} \subseteq F$ and $K$ Galois over $F$ with abelian Galois group with base $\{\rho, \sigma\}$ where $\operatorname{ord}(\rho)=r$ and $\operatorname{ord}(\sigma)=s$. (Further conditions on $F$ and $K$ will be imposed below.) Let $L_{1}$ be the fixed field of $\rho$ and $L_{2}$ the fixed field of $\sigma$. So, $\left[K: L_{1}\right]=\left[L_{2}: F\right]=r$ and $\left[K: L_{2}\right]=\left[L_{1}: F\right]=s$. Let $h \in K^{*}$ be any element with $N_{K / F}(h)=1$, where $N_{K / F}$ denotes the norm from $K$ to $F$. Then, let $D=K((i))((j))$, the iterated twisted Laurent series division algebra, where the multiplication on $D$ is specified by the relations

$$
i c=\rho(c) i, \quad j c=\sigma(c) j \quad \text { for all } c \in K, \text { and } i j=h j i,
$$

that is, $D$ is the iterated twisted Laurent series division algebra

$$
D=K((i ; \rho))\left(\left(j ; \sigma^{*}\right)\right)
$$

in the notation of $\left[\mathbf{J}_{\mathbf{2}}\right.$, p. 87], where $\sigma^{*}$ is the extension of $\sigma$ to $K((i ; \rho))$ given by $\sigma^{*}(i)=h^{-1} i$. Clearly $K((i ; \rho))$ has center $L_{1}\left(\left(i^{r}\right)\right)$ and $\left[K((i ; \rho)): L_{1}\left(\left(i^{r}\right)\right)\right]=$ $r^{2}$. We have $\sigma^{*}\left(i^{r}\right)=\left(h^{-1} i\right)^{r}=N_{K / L_{1}}\left(h^{-1}\right) i^{r}$. Since $N_{L_{1} / F}\left(N_{K / L_{1}}\left(h^{-1}\right)\right)=$ $N_{K / F}\left(h^{-1}\right)=1$, by Hilbert 90 there is an $a \in L_{1}^{*}$ with $\sigma(a) / a=N_{K / L_{1}}\left(h^{-1}\right)$. Then, as an automorphism of $L_{1}\left(\left(i^{r}\right)\right) \sigma^{*}$ has fixed field $F\left(\left(a^{-1} i^{r}\right)\right)$ and order $s$. Note that $\sigma^{* s}(i)=N_{K / L_{2}}\left(h^{-1}\right) i$. Again by Hilbert 90 there is a $b \in L_{2}^{*}$ with $N_{K / L_{2}}\left(h^{-1}\right)=b / \rho(b)$. Then $\sigma^{* s}$ agrees with conjugation by $b$ on $K((i ; \rho))$. Consequently, by $\left[\mathbf{J}_{\mathbf{2}}\right.$, Theorem 5, p. 88], the center of $D$ is $E:=F\left(\left(a^{-1} i^{r}\right)\right)\left(\left(b^{-1} j^{s}\right)\right)$, and $[D: E]=(r s)^{2}$.

(Note: There are other ways of viewing $D$ and $E$. One can check that $D$ is a Malcev-Neumann algebra, as discussed in $\left[\mathbf{T}_{\mathbf{1}}, \S 2\right]$ and $\left[\mathbf{T A}_{\mathbf{2}}\right]$. Also, one could start from the field $E=F((x))((y))$, extend $\rho$ and $\sigma$ to $K((x))((y))$ by $\rho(x)=\sigma(x)=x$ and $\rho(y)=\sigma(y)=y$, and define $D$ to be the $E$-algebra generated over $K((x))((y))$ by $i$ and $j$ subject to the relations

$$
\begin{aligned}
& i c=\rho(c) i, \quad j c=\sigma(c) j \quad \text { for all } c \in K((x))((y)), \\
& i^{r}=a x, \quad j^{s}=b y, \quad \text { and } \quad i j=h j i .
\end{aligned}
$$


From this description it is apparent that $D$ is a power series version of the generic abelian crossed product of Amitsur and Saltman [AS, §2].)

There is a standard valuation $v: D^{*} \rightarrow \mathbf{Z} \times \mathbf{Z}$ on $D$ given by

$$
v\left(\sum_{m} \sum_{n} c_{m, n} i^{m} j^{n}\right)=\inf \left\{(m, n) \mid c_{m, n} \neq 0\right\}, \quad \text { for any } c_{m, n} \in K
$$

where $\mathbf{Z} \times \mathbf{Z}$ has the right-to-left lexicographical order. This is analogous to (3.6) for fields. It is easy to check that $v$ is still a valuation, even though the multiplication on $D$ is twisted by the relations (5.2). For this valuation, we have $\Gamma_{D, v}=\mathbf{Z} \times \mathbf{Z}$, $\bar{D}_{v}=K$, and $v(i)=(1,0), v(j)=(0,1)$. The restriction of $v$ to $E$ is Henselian with $\Gamma_{E, v}=r \mathbf{Z} \times s \mathbf{Z}$ and $\bar{E}_{v}=F$.

Now, suppose $F$ has a valuation $w$ with a (unique) totally ramified extension to $K$, with $\operatorname{char}\left(\bar{F}_{w}\right) \nmid r s$. Then, as $\mu_{r s} \subseteq F, \bar{F}_{w}$ contains a primitive $r s$ root of unity $\varepsilon$. Also, since $N_{K / F}(h)=1$ and $K / F$ is totally ramified, we must have $w(h)=0$ and, in $\bar{K}_{w}, \bar{h}^{r s}=\overline{N_{K / F}(h)}=1$. Hence,

$$
\text { in } \bar{K}_{w}, \quad \bar{h}=\varepsilon^{t} \quad \text { for some } t, 1 \leq t \leq r s .
$$

Because $w$ extends uniquely from $F$ to $K$, every $F$-automorphism of $K$ preserves $w$. Therefore, if $\pi: V_{D, v} \rightarrow \bar{D}_{v}$ is the natural projection, $\pi^{-1}\left(V_{K, w}\right)$ is mapped to itself by all inner automorphisms of $D$. Since, in addition, $\pi^{-1}\left(V_{K, w}\right)$ contains $a$ or $a^{-1}$ for each $a \in D^{*}, \pi^{-1}\left(V_{K, w}\right)$ is a valuation $\operatorname{ring}$ of $D$ (cf. [S, p. 17, Theorem 6]). Let $u$ be the corresponding valuation on $D$. (Explicitly, $u\left(\sum_{m} \sum_{n} c_{m, n} i^{m} j^{n}\right)=$ $\inf \left\{\left(w\left(c_{m, n}\right),(m, n)\right) \mid c_{m, n} \neq 0\right\} \in \Gamma_{K, w} \times \Gamma_{D, v}$, where this product of value groups is ordered lexicographically right-to-left.) The usual exact sequence of value groups for composing valuations is valid here:

$$
0 \rightarrow \Gamma_{K, w} \rightarrow \Gamma_{D, u} \rightarrow \Gamma_{D, v} \rightarrow 0 \text {. }
$$

LEMMA 5.6. With respect to the valuation $u, D$ is totally ramified over $E$. Further,

$$
\Gamma_{D, u} / \Gamma_{E, u} \cong(\mathbf{Z} / l \mathbf{Z})^{2} \times(\mathbf{Z} / d \mathbf{Z})^{2}
$$

where $l=\operatorname{lcm}(r s, r t, s t) / t$ (with $t$ as in (5.4)) and $d=r s / l$.

PROOF. $u$ restricts to a valuation on the field $E \cdot K=K\left(\left(a^{-1} i^{r}\right)\right)\left(\left(b^{-1} j^{s}\right)\right)$ and to one on $E$; in each case $u$ is the composite valuation of the restrictions of $v$ and $w$. Consider the exact sequence

$$
0 \rightarrow \Gamma_{E \cdot K, u} / \Gamma_{E, u} \rightarrow \Gamma_{D, u} / \Gamma_{E, u} \rightarrow \Gamma_{D, u} / \Gamma_{E \cdot K, u} \rightarrow 0
$$

Since $\Gamma_{E \cdot K, v}=\Gamma_{E, v}$, we have $\Gamma_{E \cdot K, u} / \Gamma_{E, u} \cong \Gamma_{K, w} / \Gamma_{F, w}$ (as one can see by comparing the exact sequences (5.5) for $E \cdot K$ and $E$ ); likewise, since $\bar{D}_{v}=K=\bar{E}_{\bar{v}}$, we have $\Gamma_{D, u} / \Gamma_{E \cdot K, u} \cong \Gamma_{D, v} / \Gamma_{E \cdot K, v}$. Thus, with respect to $u, D$ is totally ramified over $E \cdot K$ and $E \cdot K$ is totally ramified over $E$; hence $D$ is totally ramified over $E$. Let $G=\Gamma_{D, u} / \Gamma_{E, u}$. Writing $\bar{u}$ for the composite map $D^{*} \rightarrow \Gamma_{D, u} \rightarrow \Gamma_{D, u} / \Gamma_{E, u}$, we see from (5.7) that $G$ is generated by $\bar{u}(i), \bar{u}(j)$, and the subgroup $H:=\Gamma_{E \cdot K, u} / \Gamma_{E, u}$. Note also that $r \bar{u}(i), s \bar{u}(j) \in H$ as $i^{r}, j^{s} \in E \cdot K$. The canonical pairing $C_{D}$ for $u$ on $D$ is completely determined by the conditions:

$$
\begin{aligned}
& C_{D}(\bar{u}(i), \bar{u}(c))=\overline{\rho(c) / c}, \quad C_{D}(\bar{u}(j), \bar{u}(c))=\overline{\sigma(c) / c} \text { for all } c \in K^{*}, \\
& C_{D}(\bar{u}(i), \bar{u}(j))=\bar{h}=\varepsilon^{t}, \\
& H \text { is a Lagrangian (as } E \cdot K \text { is a field). }
\end{aligned}
$$


Now, $\varepsilon^{s}$ is a primitive $r$ th root of unity. By Kummer theory (or by invoking (1.4) above) there is an $\alpha \in \bar{u}\left(L_{2}^{*}\right)$ with $C_{D}(\bar{u}(i), \alpha)=\varepsilon^{s}$. So $r \mid \operatorname{ord}(\alpha)$. But, as $\Gamma_{E \cdot L_{2}, u} / \Gamma_{E, u} \cong \mathcal{G}\left(E \cdot L_{2} / E\right) \cong \mathcal{G}\left(L_{2} / F\right)$ which has order $r$, ord $(\alpha)=r$. Likewise, there is a $\beta \in \bar{u}\left(L_{1}^{*}\right)$ with $C_{D}(\bar{u}(j), \beta)=\varepsilon^{r}$, and $\operatorname{ord}(\beta)=s$. We have

$$
\begin{aligned}
& C_{D}(\bar{u}(i), \alpha)=\varepsilon^{s}, \quad C_{D}(\bar{u}(j), \alpha)=1 \quad\left(\text { as } \rho \text { stabilizes } L_{2}\right), \\
& C_{D}(\bar{u}(i), \beta)=1, \quad C_{D}(\bar{u}(j), \beta)=\varepsilon^{r}, \\
& C_{D}(\alpha, \beta)=1, \quad C_{D}(\bar{u}(i), \bar{u}(j))=\varepsilon^{t} .
\end{aligned}
$$

From these formulas it follows $\langle\alpha\rangle \cap\langle\beta\rangle=1$, so $\{\alpha, \beta\}$ is a base of the abelian group $H$. Furthermore, if we write $r \bar{u}(i)=n_{1} \alpha+m_{1} \beta$ in $H$, we have $1=C_{D}(\bar{u}(i), r \bar{u}(i))=$ $\varepsilon^{n_{1} s}$, so $r \mid n_{1}$ and $n_{1} \alpha=0$. Thus,

$$
r \bar{u}(i)=m_{1} \beta \quad \text { and, likewise, } \quad s \bar{u}(j)=m_{2} \alpha,
$$

for some integers $m_{1}$ and $m_{2}$. Hence, $G=G_{1} \times G_{2}$, where $G_{1}=\langle\beta, \bar{u}(i)\rangle$ and $G_{2}=\langle\alpha, \bar{u}(j)\rangle$. Note that

$$
\begin{aligned}
& \varepsilon^{r t}=C_{D}(r \bar{u}(i), \bar{u}(j))=C_{D}\left(m_{1} \beta, \bar{u}(j)\right)=\varepsilon^{-m_{1} r} \\
& \varepsilon^{s t}=C_{D}(\bar{u}(i), s \bar{u}(j))=C_{D}\left(\bar{u}(i), m_{2} \alpha\right)=\varepsilon^{m_{2} s}
\end{aligned}
$$

so $m_{1} \equiv-t(\bmod s)$ and $m_{2} \equiv t(\bmod r)$. Thus, $\operatorname{gcd}\left(s, m_{1}\right)=\operatorname{gcd}(s, t)$ and $\operatorname{gcd}\left(r, m_{2}\right)=\operatorname{gcd}(r, t)$.

Consider the group $G_{1}=\langle\beta, \bar{u}(i)\rangle$. Since the image of $\bar{u}(i)$ has order $r$ in $G_{1} /\langle\beta\rangle$, $\left|G_{1}\right|=r s$ and

$$
\begin{aligned}
\operatorname{ord}(\bar{u}(i)) & =r \operatorname{ord}(r \bar{u}(i))=r \operatorname{ord}\left(m_{1} \beta\right)=r s / \operatorname{gcd}\left(s, m_{1}\right) \\
& =r s / \operatorname{gcd}(s, t)=r \operatorname{lcm}(s, t) / t .
\end{aligned}
$$

Therefore, $G_{1}$ has exponent

$$
\operatorname{lcm}(\operatorname{ord}(\beta), \operatorname{ord} \bar{u}(i))=\operatorname{lcm}(s, r \operatorname{lcm}(s, t) / t)=\operatorname{lcm}(s t, r s, r t) / t=l .
$$

Since the abelian group $G_{1}$ has two generators its rank is at most 2 ; thus, its invariant factors are determined by its order and exponent. That is, $G_{1} \cong(\mathbf{Z} / l \mathbf{Z}) \times$ $(\mathbf{Z} / d \mathbf{Z})$, where $d=\left|G_{1}\right| / \exp \left(G_{1}\right)=r s / l$. An analogous calculation shows that $G_{2}$ has the same order and exponent as $G_{1}$. Thus,

$$
G=G_{1} \times G_{2} \cong G_{1} \times G_{1} \cong(\mathbf{Z} / l \mathbf{Z})^{2} \times(\mathbf{Z} / d \mathbf{Z})^{2},
$$

as desired.

An interesting feature of this lemma is the way the relative value group depends on $\bar{h}$ as well as $r$ and $s$.

We now construct fields $F$ and $K$ so that the lemma can be applied in more than one way. Let $k$ be any field containing a primitive $r s$-root of unity $\varepsilon$, so $\operatorname{char}(k) \nmid r s$. Let $F_{0}=k\left(z_{1}, z_{2}\right)$ and $K_{0}=k\left(z_{1}^{1 / r}, z_{2}^{1 / s}\right)$, where $z_{1}$ and $z_{2}$ are algebraically independent over $k$. Let $w$ be the valuation obtained by restricting to $F_{0}$ the standard valuation on $k\left(\left(z_{1}\right)\right)\left(\left(z_{2}\right)\right)$. Note that $w$ has a totally ramified, hence unique, extension to $K_{0}$. We enlarge $F_{0}$ and $K_{0}$ to get more valuations and to obtain a convenient element of norm 1 . Let

$$
\delta=N_{K_{0} / F_{0}}\left(1-z_{1}^{1 / r}-z_{2}^{1 / s}\right)=\prod_{m=1}^{r} \prod_{n=1}^{s}\left(1-\varepsilon^{s m} z_{1}^{1 / r}-\varepsilon^{r n} z_{2}^{1 / s}\right) \in k\left[z_{1}, z_{2}\right] .
$$


Note that since $\delta$ factors in $k\left[z_{1}^{1 / r}, z_{2}^{1 / s}\right]$ into a product of $r s$ distinct (and nonassociate) irreducibles, every irreducible factor of $\delta$ in $k\left[z_{1}, z_{2}\right]$ occurs with multiplicity 1. (In fact, one can check that $\delta$ is irreducible in $k\left[z_{1}, z_{2}\right]$.)

Let $\gamma=\delta^{1 / r s}$, and let $F=F_{0}(\gamma), K=K_{0}(\gamma)=K_{0} \cdot F$. It is clear from the prime factorization of $\delta$ that $\left[F: F_{0}\right]=r s$. Since $\delta$ is a 1-unit of $\left(F_{0}, w\right), w$ has $r s$ different extensions $w_{1}, \ldots, w_{r s}$ to $F$, and the images of $\gamma$ in the residue fields $\bar{F}_{w_{m}}$ range over the powers of $\varepsilon$; number the $w_{m}$ so that $\bar{\gamma}=\varepsilon^{m}$ in $\bar{F}_{w_{m}}, 1 \leq m \leq r s$. Also, $K_{0}$ is linearly disjoint to $F$ over $F_{0}$, as $\left(K_{0}, w\right)$ is totally ramified over $\left(F_{0}, w\right)$ while each $\left(F, w_{m}\right)$ is an immediate extension of $\left(F_{0}, w\right)$. Hence $[K: F]=r s, K$ is Galois over $F$ with $\mathcal{G}(K / F) \cong \mathcal{G}\left(K_{0} / F_{0}\right) \cong(\mathbf{Z} / r \mathbf{Z}) \times(\mathbf{Z} / s \mathbf{Z})$, and each $w_{m}$ has a totally ramified (hence unique) extension to $K$. Let $\{\rho, \sigma\}$ be any base of $\mathcal{G}(K / F)$ with $\operatorname{ord}(\rho)=r$ and $\operatorname{ord}(\sigma)=s$. Let

$$
h=\gamma /\left(1-z_{1}^{1 / r}-z_{2}^{1 / s}\right) \in K
$$

Then, $N_{K / F}(h)=\gamma^{r s} / N_{K_{0} / F_{0}}\left(1-z_{1}^{1 / r}-z_{2}^{1 / s}\right)=\delta / \delta=1$.

PROPOSITION 5.8. Let $D$ be the division algebra constructed as in (5.2) and (5.3) from the $K, F, \rho, \sigma$, and $h$ just defined. Then, if $\operatorname{gcd}(r, s)>1, D$ is not a symbol algebra, nor is $D$ isomorphic to a tensor product of symbol algebras.

PROOF. For each $m$ with $1 \leq m \leq r s$, we can apply Lemma 5.6 with $w_{m}$ for $w$, obtaining a valuation $u_{m}$ on $D$ which is totally ramified over $Z(D)=E$. Since $\bar{h}=\varepsilon^{m} / 1=\varepsilon^{m}$ in $\bar{K}_{w_{m}}$, we have $t=m$ in (5.4). Hence, the lemma shows $\Gamma_{D, u_{1}} / \Gamma_{E, u_{1}} \cong(\mathbf{Z} / r s \mathbf{Z})^{2}$, while for any prime $p$ dividing both $r$ and $s$, $\Gamma_{D, u_{p}} / \Gamma_{E, u_{p}} \cong(\mathbf{Z} /(r s / p) \mathbf{Z})^{2} \times(\mathbf{Z} / p \mathbf{Z})^{2}$. If $D$ were a symbol algebra or a tensor product of such algebras, then it would have an armature $D$. Two applications of $(3.3)$ show that $D \cong(\mathbf{Z} / r s \mathbf{Z})^{2}$ and $D \cong(\mathbf{Z} /(r s / p) \mathbf{Z})^{2} \times(\mathbf{Z} / p \mathbf{Z})^{2}$, a contradiction.

REMARKS 5.9. (i) For the $D$ in (5.8), $[D: E]=(r s)^{2}$, and Proposition 4.7(i) with the valuation $u_{1}$ shows that ord $[D]=r s$ in $\operatorname{Br}(F)$. Also, an application of $(4.7)(\mathrm{v})$ with the valuation $u_{p}$ shows that $D$ is not split by any cyclic Galois extension of $E$. As $m$ ranges between 1 and $r s$, the relative value groups $\Gamma_{D, u_{m}} / \Gamma_{F, u_{m}}$ range over all the groups described in (5.6), for all values of $t$. These are precisely all the abelian groups which admit a nondegenerate symplectic pairing and have a Lagrangian isomorphic to $(\mathbf{Z} / r \mathbf{Z}) \times(\mathbf{Z} / s \mathbf{Z})$. (When $\operatorname{gcd}(r, s)=1,(\mathbf{Z} / r \mathbf{Z})^{2} \times(\mathbf{Z} / s \mathbf{Z})^{2}$ is the only such group, but when $r$ and $s$ have a common factor there is more than one such group - that is what was needed for (5.8).) The smallest dimensional division algebra to which (5.8) applies occurs when $r=s=2$, so that $[D: F]=16$.

(ii) The $D$ in Proposition 5.8 is totally ramified over $Z(D)=E$ with respect to each of the valuations $u_{m}$, which are not Henselian on $E$. The proposition shows that the Henselian hypothesis in Draxl's decomposition Theorem 3.7 cannot be dropped.

(iii) Proposition 5.8 also shows that a division algebra $D$ over a field with Henselian valuation need not be isomorphic to a product of symbol algebras even if $\bar{D}$ is a field. For this use the Henselian valuation $v$ on $E$, for which $\bar{D}_{v}=K$. In addition, if $r$ and $s$ have the same prime factors, it can be shown that there are no totally ramified field extensions of $(E, v)$ in $D$, and that no totally ramified algebraic field extension of $(E, v)$ splits $D$. 


\section{REFERENCES}

[A 1 ] A. A. Albert, On the Wedderburn norm condition for cyclic algebras, Bull. Amer. Math. Soc. 37 (1931), 301-312.

$\left[\mathbf{A}_{2}\right] \ldots$, Noncyclic algebras of degree and exponent four, Trans. Amer. Math. Soc. 35 (1933), 112121.

[A $\mathbf{A}]$ _ Structure of algebras, Amer. Math. Soc. Colloq. Publ., vol. 24, Amer. Math. Soc., Providence, R. I., 1961.

[Am] S. A. Amitsur, On central division algebras, Israel J. Math. 12 (1972), 408-420.

[AS] S. A. Amitsur and D. Saltman, Generic abelian crossed products and p-algebras, J. Algebra 51 (1978), 76-87.

[B] N. Bourbaki, Algèbre commutative, Chapitre VI, Valuations, Hermann, Paris, 1961.

[C] P. M. Cohn, On extending valuations in division algebras, Studia Sci. Math. Hungar. 16 (1981), 65-70.

[CM] P. M. Cohn and M. Mahdavi-Hezavehi, Extensions of valuations on skew fields, Ring Theory, Antwerp 1980 (F. Van Oystaeyen, ed.), Lecture Notes in Math., vol. 825, Springer-Verlag, Berlin and New York, 1980, pp. 28-41.

[D $]$ P. Draxl, Skew fields, London Math. Soc. Lecture Note Series, no. 81, Carnbridge Univ. Press, Cambridge, 1983.

[D2] _ Ostrowski's theorem for Henselian valued skew fields, J. Reine Angew. Math. 354 (1984), 213-218.

[DK] P. Draxl and M. Kneser (eds.), SK 1 von Schiefkörpern, Lecture Notes in Math., vol. 778, Springer-Verlag, Berlin and New York, 1980.

[E] O. Endler, Valuation theory, Springer-Verlag, New York, 1972.

[H] H. Hasse, Über p-adische Schiefkörper und ihre Bedeutung für die Arithmetik hyperkomplexer Zahlsysteme, Math. Ann. 104 (1931), 495-534.

[JW] B. Jacob and A. Wadsworth, A new construction of noncrossed product algebras, Trans. Amer. Math. Soc. 293 (1986), 693-721.

[J $\left.\mathbf{J}_{1}\right]$ N. Jacobson, Lectures in abstract algebra, Vol. III, Van Nostrand, Princeton, N.J., 1964.

$\left[\mathbf{J}_{\mathbf{2}}\right] \ldots$, P. I.-algebras, an introduction, Lecture Notes in Math., vol. 441, Springer-Verlag, Berlin and New York, 1975.

[M] J. Milnor, Introduction to algebraic K-theory, Ann. of Math. Studies, No. 72, Princeton Univ. Press, Princeton, N. J., 1971.

[P] V. P. Platonov, The Tannaka-Artin problem and reduced $K$-theory, Izv. Akad. Nauk SSSR Ser. Mat. 40 (1976), 227-261; English transl. in Math. USSR Izv. 10 (1976), 211-243.

[PY] V. P. Platonov and V. I. Yanchevskii, Dieudonné's conjecture on the structure of unitary groups, and division rings over Henselian fields, Dokl. Akad. Nauk SSSR 279 (1984), 546-549; English transl. in Soviet Math. Dokl. 30 (1984), 693-696.

[Ri] P. Ribenboim, Théorie des valuations, Presses Univ. Montréal, Montréal, 1968.

[Ro] J. Rotman, Introduction to the theory of groups, 3rd ed., Allyn and Bacon, Boston, Mass., 1984.

[Sa] D. Saltman, Indecomposable division algebras, Comm. Algebra 7 (1979), 791-817.

[Sch] W. Scharlau, Über die Brauer-Gruppe eines Henselkörpers, Abh. Math. Sem. Univ. Hamburg 33 (1969), 243-249.

[S] O. F. G. Schilling, The theory of valuations, Math. Surveys, no. 4, Amer. Math. Soc., Providence, R. I., 1950.

[Ta] J. Tate, Relations between $K_{2}$ and Galois cohomology, Invent. Math. 36 (1976), 257-274.

[ $\left.\mathbf{T}_{\mathbf{1}}\right]$ J.-P. Tignol, Sur les décompositions des algèbres à division en produit tensoriel d'algèbres cycliques, Brauer Groups in Ring Theory and Algebraic Geometry (F. Van Oystaeyen and A. Verschoren, eds.) Lecture Notes in Math., vol. 917, Springer-Verlag, Berlin and New York, 1982, pp. 126-145.

$\left[\mathbf{T}_{\mathbf{2}}\right] \ldots$, On the length of decompositions of central simple algebras in tensor products of symbols, Methods in Ring Theory (F. Van Oystaeyen, ed.), NATO ASI Series, Series C, Vol. 129, Reidel, Dordrecht, 1984, pp. 505-516.

[TA 1 ] J.-P. Tignol and S. A. Amitsur, Totally ramified splitting fields of central simple algebras over Henselian fields, J. Algebra 98 (1986), 95-101.

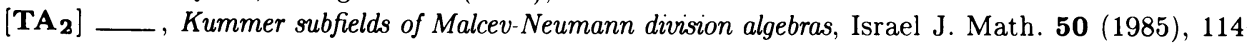
144. 
[TA $\mathbf{T}]$, Symplectic modules, Israel J. Math. 54 (1986), 266-290.

[VG] J. Van Geel, Places and valuations in noncommutative ring theory, Lecture Notes in Pure and Appl. Math., no. 71, Marcel Dekker, New York, 1981.

[W $\mathbf{W}_{1}$ A. Wadsworth, p-Henselian fields: K-theory, Galois cohomology, and Witt rings, Pacific J. Math. 105 (1983), 473-495.

[ $\left.\mathbf{W}_{\mathbf{2}}\right]$ _ Extending valuations to finite-dimensional division algebras, Proc. Amer. Math. Soc. 98 (1986), 20-22.

DÉpartement de Mathématique, Université Catholique de Louvain, B-1348 LOUVAIN-LA-NEUVE, BELGIUM

Department of Mathematics, University of California, San Diego, la Jolla, CALIFORNIA 92093 